\title{
Computational Approaches to Lattice Packing and Covering Problems*
}

\author{
Achill Schürmann ${ }^{1}$ and Frank Vallentin ${ }^{2}$ \\ ${ }^{1}$ Department of Mathematics, University of Magdeburg, \\ 39106 Magdeburg, Germany \\ achill@math.uni-magdeburg.de \\ ${ }^{2}$ Einstein Institute of Mathematics, The Hebrew University of Jerusalem, \\ Jerusalem 91904, Israel \\ frank.vallentin@gmail.com
}

\begin{abstract}
We describe algorithms which address two classical problems in lattice geometry: the lattice covering and the simultaneous lattice packing-covering problem. Theoretically our algorithms solve the two problems in any fixed dimension $d$ in the sense that they approximate optimal covering lattices and optimal packing-covering lattices within any desired accuracy. Both algorithms involve semidefinite programming and are based on Voronoi's reduction theory for positive definite quadratic forms, which describes all possible Delone triangulations of $\mathbb{Z}^{d}$.

In practice, our implementations reproduce known results in dimensions $d \leq 5$ and in particular solve the two problems in these dimensions. For $d=6$ our computations produce new best known covering as well as packing-covering lattices, which are closely related to the lattice $\mathrm{E}_{6}^{*}$. For $d=7,8$ our approach leads to new best known covering lattices. Although we use numerical methods, we made some effort to transform numerical evidences into rigorous proofs. We provide rigorous error bounds and prove that some of the new lattices are locally optimal.
\end{abstract}

\section{Overview}

Two classical problems in the geometry of numbers are the determination of the most economical lattice sphere packings and coverings of the Euclidean $d$-space $\mathbb{E}^{d}$. In this

* The work by Frank Vallentin was partially supported by the Edmund Landau Center for Research in Mathematical Analysis and Related Areas, sponsored by the Minerva Foundation (Germany). Both authors were supported by the "Deutsche Forschungsgemeinschaft" (DFG) under Grant SCHU 1503/4-1. 
paper we describe algorithms for the lattice covering and the simultaneous lattice packing and covering problem (lattice packing-covering problem in what follows).

Roughly speaking, both problems are concerned with the most economical way to cover $\mathbb{E}^{d}$. In the case of the lattice covering problem, the goal is to maximize the volume of a fundamental domain in a lattice covering with unit spheres. Roughly speaking, we want to minimize the number of unit spheres which are needed to cover arbitrarily large but finite regions of $\mathbb{E}^{d}$. The objective of the lattice packing-covering problem is to maximize the minimal distance between lattice points in a lattice covering with unit spheres.

The aim of this paper is to give an introduction to the mathematical tools that allow us, at least in theory, to solve the two problems computationally. For a fixed dimension $d$, our algorithms approximate optimal covering lattices and optimal packing-covering lattices within any desired accuracy. In this overview we describe the structure of the paper.

The basic concepts and notations, which we use throughout the paper, are in Section 2. There we also give a precise definition of the two problems under consideration. The reader familiar with sphere packings and coverings, as well as lattices and their relation to positive definite quadratic forms, may skip this section.

In Section 3 we review known results and the history of the lattice covering problem, and in Section 4 we review known results and the history of the packing-covering problem.

Our algorithms as well as the known results by other authors are mainly based on a reduction theory for positive definite quadratic forms by Voronoi. We give a detailed description of this main ingredient in Section 5 with a special focus on computational implementability.

The other main tool comes from convex optimization theory. Semidefinite programming problems and determinant maximization problems are briefly described in Section 6 . We describe how duality theory together with rational approximations can be used to provide rigorous error bounds. Both problems have in common that one has to minimize a convex function on variables that satisfy some linear matrix inequalities (LMIs).

In Section 7 we describe how the constraint that a lattice gives a unit sphere covering can be modeled by LMIs.

In Section 8 we combine these tools and attain algorithms which theoretically solve the two problems under consideration.

Due to a combinatorial explosion of the number of different Delone triangulations, our implementations of the algorithms only give complete solutions for $d \leq 5$. Moreover, the convex optimization algorithms we used are interior point methods and so yield only approximations. Therefore, in Section 9, we collect some mathematical tools which allow us to determine exact results from these approximations. In particular we can test computationally whether or not a given positive definite quadratic form gives a locally optimal solution. In the case of the lattice packing-covering problem, we can test if such a solution is isolated.

In order to run a heuristic search for good lattices, it is necessary to have local lower bounds that we can compute fast. We describe one class of such bounds depending on the methods of inertia in Section 10, which we used to find new lattices in dimension 6. 
Both problems have been previously solved only for dimensions $d \leq 5$. Our implementations not only verify all of these results, but also attain additional information on locally optimal solutions for $d=5$. Moreover, we find new best known lattices for both problems in dimension 6, 7 and 8. In particular, we answer an open question by Ryshkov. In Section 11 we report on our results. There we distinguish between conjectures for which we only have numerical evidence and theorems for which we have rigorous proofs.

\section{Basic Concepts and Notations}

In this section we fix the notation we use throughout this paper. We refer the reader to [13], [41] and [22] for further information about the introduced concepts. The reader familiar with sphere packings and coverings, as well as lattices and their relation to positive definite quadratic forms, may skip this section.

Let $\mathbb{E}^{d}$ be a $d$-dimensional Euclidean space equipped with inner product $\langle\cdot, \cdot\rangle$, norm $\|\cdot\|$ and unit ball $B^{d}=\left\{\boldsymbol{x} \in \mathbb{E}^{d}:\|\boldsymbol{x}\| \leq 1\right\}$. A lattice $L$ is a discrete subgroup in $\mathbb{E}^{d}$. From now on we assume that all lattices $L$ have full rank $d$; that is, there exists a regular matrix $A \in \mathrm{GL}_{d}(\mathbb{R})$ with $L=A \mathbb{Z}^{d}$. The columns of the matrix $A$ are called a basis of $L$. All bases of $L$ are of the form $A U$ with $U \in \mathrm{GL}_{d}(\mathbb{Z})$. Thus, the determinant $\operatorname{det}(L)=|\operatorname{det}(A)|>0$ of the lattice $L$ is well defined. We say that two lattices $L$ and $L^{\prime}$ belong to the same isometry class if, for every basis $A$ of $L$, there is a basis $A^{\prime}$ of $L^{\prime}$ and an orthogonal transformation $O \in \mathrm{O}_{d}(\mathbb{R})$ such that $A^{\prime}=O A$.

The Minkowski sum $L+\alpha B^{d}=\left\{\boldsymbol{v}+\alpha \boldsymbol{x}: \boldsymbol{v} \in L, \boldsymbol{x} \in B^{d}\right\}$, with $\alpha \in \mathbb{R}_{>0}$, is a lattice packing if the translates of $\alpha B^{d}$ have mutually disjoint interiors and a lattice covering if $\mathbb{E}^{d}=L+\alpha B^{d}$. The packing radius $\lambda(L)$ of a lattice $L$ is given by

$$
\lambda(L)=\max \left\{\lambda: L+\lambda B^{d} \text { is a lattice packing }\right\}
$$

and the covering radius $\mu(L)$ by

$$
\mu(L)=\min \left\{\mu: L+\mu B^{d} \text { is a lattice covering }\right\} .
$$

The above values are attained: The packing radius is equal to half the length of a shortest non-zero vector of $L$ and the covering radius is equal to the maximum distance of points in $\mathbb{E}^{d}$ to a closest lattice vector. The packing radius is the inradius of the Dirichlet-Voronoi polytope of $L$,

$$
\mathrm{DV}(L)=\left\{\boldsymbol{x} \in \mathbb{E}^{d}:\|\boldsymbol{x}\| \leq\|\boldsymbol{x}-\boldsymbol{v}\|, \boldsymbol{v} \in L\right\},
$$

and the covering radius is its circumradius. Both functionals are homogeneous, that is, for $\alpha \in \mathbb{R}$ we have

$$
\mu(\alpha L)=|\alpha| \mu(L) \quad \text { and } \quad \lambda(\alpha L)=|\alpha| \lambda(L) .
$$

Thus, the covering density

$$
\Theta(L)=\frac{\mu(L)^{d}}{\operatorname{det}(L)} \cdot \kappa_{d}, \quad \kappa_{d}=\operatorname{vol} B^{d},
$$


is invariant with respect to scaling of $L$. The same is true for the packing-covering constant

$$
\gamma(L)=\frac{\mu(L)}{\lambda(L)} .
$$

All these functionals are invariants of the isometry classes.

In this paper we study the following two problems:

Problem 2.1 (Lattice Covering Problem). For a given $d \in \mathbb{N}$, determine $\Theta_{d}=$ $\min _{L} \Theta(L)$, where $L \subseteq \mathbb{E}^{d}$ runs over all $d$-dimensional lattices.

Problem 2.2 (Lattice Packing-Covering Problem). For a given $d \in \mathbb{N}$, determine $\gamma_{d}=$ $\min _{L} \gamma(L)$, where $L \subseteq \mathbb{E}^{d}$ runs over all $d$-dimensional lattices.

We describe the history and results of both problems in Sections 3 and 4. There we assume that the reader is familiar with certain important lattices which are described in Chapter 4 of [22]. Historically and for computational reasons, the problems were studied by using the intimate relation between lattices and positive definite quadratic forms (PQFs).

We describe this relation: To a $d$-dimensional lattice $L=A \mathbb{Z}^{d}$ with basis $A$ we associate a $d$-dimensional PQF

$$
Q[x]=x^{t} A^{t} A x=x^{t} G x,
$$

where the Gram matrix $G=A^{t} A$ is symmetric and positive definite. We abuse notation and identify quadratic forms with symmetric matrices by saying $Q=G$ and $Q[x]=$ $\boldsymbol{x}^{t} Q \boldsymbol{x}$. The set of quadratic forms is a $(d(d+1) / 2)$-dimensional real vector space $\mathcal{S}^{d}$, in which the set of PQFs forms an open, convex cone $\mathcal{S}_{>0}^{d}$. Its closure is the convex cone of all positive semidefinite quadratic forms $\mathcal{S}_{\geq 0}^{d}$, which is pointed at $\boldsymbol{O}$.

Note that $Q$ depends on the chosen basis $A$ of $L$. Two arbitrary bases $A$ and $B$ of $L$ are transformed into each other by a unimodular transformation, that is, there exists a $U \in \mathrm{GL}_{d}(\mathbb{Z})$ such that $A=B U$. Thus, $\mathrm{GL}_{d}(\mathbb{Z})$ acts on $\mathcal{S}_{>0}^{d}$ by $Q \mapsto U^{t} Q U$. Two $\mathrm{PQFs}$ lying in the same orbit under this action are called arithmetically equivalent. This definition naturally extends to positive semidefinite quadratic forms.

Thus every lattice uniquely determines an arithmetical equivalence class of PQFs. On the other hand, every PQF $Q$ admits a Cholesky decomposition $Q=A^{t} A$, where the upper triangular matrix $A$ is uniquely determined up to an orthogonal transformation $O \in \mathrm{O}_{d}(\mathbb{R})$. Altogether, we have a bijection between isometry classes of lattices $\mathrm{O}_{d}(\mathbb{R}) \backslash \mathrm{GL}_{d}(\mathbb{R}) / \mathrm{GL}_{d}(\mathbb{Z})$ and arithmetical equivalence classes of PQFs $\mathcal{S}_{>0}^{d} / \mathrm{GL}_{d}(\mathbb{Z})$.

As a consequence, the lattice covering and the lattice packing-covering problem translate into problems for $\mathrm{PQFs}$ : The determinant (or discriminant) of a $\mathrm{PQF} Q$ is $\operatorname{defined}$ by $\operatorname{det}(Q)$. The homogeneous minimum $\lambda(Q)$ and the inhomogeneous minimum $\mu(Q)$ are given by

$$
\lambda(Q)=\min _{\boldsymbol{v} \in \mathbb{Z}^{d} \backslash\{\boldsymbol{\theta}\}} Q[\boldsymbol{v}] \quad \text { and } \quad \mu(Q)=\max _{\boldsymbol{x} \in \mathbb{E}^{d}} \min _{\boldsymbol{v} \in \mathbb{Z}^{d}} Q[\boldsymbol{x}-\boldsymbol{v}]
$$




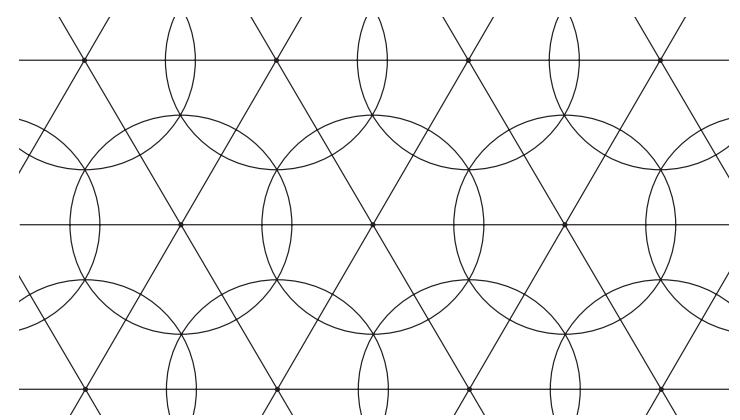

Fig. 1. The sphere covering given by the hexagonal lattice.

A corresponding lattice $L$ satisfies $\operatorname{det}(L)=\sqrt{\operatorname{det}(Q)}, \mu(L)=\sqrt{\mu(Q)}, \lambda(L)=$ $\sqrt{\lambda(Q)} / 2$. Therefore our goal is to minimize

$$
\Theta(Q)=\Theta(L)=\sqrt{\frac{\mu(Q)^{d}}{\operatorname{det} Q}} \cdot \kappa_{d} \quad \text { and } \quad \gamma(Q)=\gamma(L)=2 \cdot \sqrt{\frac{\mu(Q)}{\lambda(Q)}}
$$

among all PQFs $Q \in \mathcal{S}_{>0}^{d}$.

Since $\Theta(Q)$ and $\gamma(Q)$ are invariant with respect to the action of $\mathrm{GL}_{d}(\mathbb{Z})$ on $\mathcal{S}_{>0}^{d}$, we only need to consider one PQF in each arithmetical equivalence class. Finding a fundamental domain in $\mathcal{S}_{>0}^{d}$ is one of the most basic and classical problems in the geometry of numbers. Such a reduction theory for PQFs, especially suitable for Problems 2.1 and 2.2, is due to Voronoi. We describe it in detail in Section 5.

\section{The Lattice Covering Problem}

Kershner, in 1939, was the first to consider the lattice convering problem. In [45] he showed that the hexagonal lattice (see Fig. 1) gives the most economical sphere covering in the plane even without the restriction of being a lattice covering.

Since then the lattice covering problem has been solved up to dimension 5 (see Table 1 ). In all these cases the lattice $\mathrm{A}_{d}^{*}$, whose covering density equals

$$
\Theta\left(\mathrm{A}_{d}^{*}\right)=\sqrt{\left(\frac{d(d+2)}{12(d+1)}\right)^{d}(d+1)} \cdot \kappa_{d},
$$

Table 1. Optimal lattice coverings.

\begin{tabular}{ccc}
\hline$d$ & Lattice covering & Covering density $\Theta_{d}$ \\
\hline 1 & $\mathbb{Z}^{1}$ & 1 \\
2 & $\mathrm{~A}_{2}^{*}$ & 1.209199 \\
3 & $\mathrm{~A}_{3}^{*}$ & 1.463505 \\
4 & $\mathrm{~A}_{4}^{*}$ & 1.765529 \\
5 & $\mathrm{~A}_{5}^{*}$ & 2.124286 \\
\hline
\end{tabular}


provides the optimal lattice covering. Gameckii [38], [39] and Bleicher [18] were the first to compute the covering density of $\mathrm{A}_{d}^{*}$ for general $d$. They also showed that it is locally optimal with respect to the covering density in every dimension.

The optimality of the body centered cubic lattice $A_{3}^{*}$ whose Dirichlet-Voronoi polytope is a regular truncated octahedron (the Dirichlet-Voronoi polytope of $A_{d}^{*}$ is a regular permutahedron) was first proven by Bambah [5]. Later, Barnes substantially simplified Bambah's proof in [14] and strengthened the result by showing that in dimensions 2 and 3 the lattice $A_{d}^{*}$ is the unique locally optimal lattice covering. He used Voronoi's reduction theory and anticipated that this is the right setup for solving the lattice covering problem in dimensions larger than 3. Our algorithm in Section 8.1 confirms his anticipation. A third proof, which is mainly elementary and unlike the previous two does not use any reduction theory of PQFs, was given by Few [37]. At the moment no attempt is known to the authors to show that the optimal three-dimensional lattice covering also gives an optimal sphere covering without lattice restriction.

In [4] Bambah conjectured that the lattice $A_{4}^{*}$ gives the least dense four-dimensional lattice covering. In [28] Delone and Ryshkov proved Bambah's conjecture. In [7] and [8] Baranovskii gave an alternative proof of this fact. He determined all locally optimal lattice coverings in dimension 4. Dickson [29] gave another alternative proof of this fact.

In a series of papers [51], [11], [12], [53] Ryshkov and Baranovskii solved the lattice covering problem in dimension 5. They prepared a 140-page long monograph [54] based on their investigations.

In [50] Ryshkov raised the question of finding the lowest dimension $d$ for which there is a better lattice covering than the one given by $\mathrm{A}_{d}^{*}$. In the same paper he showed that $\mathrm{A}_{d}^{*}$ is not the most efficient lattice covering for all even $d \geq 114$ and for all odd $d \geq 201$. One of our main results in this paper is the answer to Ryshkov's question (see Theorem 11.3): In Theorem 11.3 we show that dimension $d=6$ is the lowest dimension for which there is a better lattice covering than the one given by $\mathrm{A}_{d}^{*}$. The proof is based on a computer search. In Section 8.1 we give an algorithm which finds all locally optimal lattice coverings in a given dimension. Using this algorithm we are able to verify all of the known results about optimal lattice coverings up to dimension 5. Unfortunately, due to a combinatorial explosion, the algorithm cannot be applied practically in dimension 6 or greater. Nevertheless, we are able to find good lattice coverings in dimension 6,7 and 8 by applying several heuristics. We give more details in Section 11 .

What else is known? In Table 2 we list all the least dense known lattice coverings in dimensions 6-24. At the same time this list gives the least dense known sphere coverings: There is no covering of equal spheres known which is less dense than the best known lattice covering. This table is an update of Table 2.1 in [22] and we provide an up-to-date table on our web page [58]. We conclude this section by briefly describing the origins of these updates.

The Leech lattice $\Lambda_{24}$ yields the best known lattice covering in dimension 24. The covering density of the Leech lattice was computed by Conway et al. [22, Chapter 23]. It is not too brave to conjecture that the Leech lattice gives the optimal 24-dimensional sphere covering. In [57] we took a first step towards proving this conjecture by showing that the Leech lattice gives a locally optimal lattice covering. Using the Leech lattice Bambah and Sloane constructed in [6] a series of lattices in dimensions $d \geq 24$ which give a thinner lattice covering than $\mathrm{A}_{d}^{*}$. It seems that as a "corollary" of the existence of the Leech lattice the duals of the laminated lattices $\Lambda_{22}$ and $\Lambda_{23}$ give good lattice 
Table 2. Least dense known (lattice) coverings up to dimension 24 .

\begin{tabular}{rcr}
\hline$d$ & Lattice & Covering density $\Theta$ \\
\hline 6 & $\mathrm{~L}_{6}^{c 1}$ & 2.464801 \\
7 & $\mathrm{~L}_{7}^{c}$ & 2.900024 \\
8 & $\mathrm{~L}_{8}^{c}$ & 3.142202 \\
9 & $\mathrm{~A}_{9}^{5}$ & 4.340185 \\
10 & $\mathrm{~A}_{10}^{*}$ & 5.251713 \\
11 & $\mathrm{~A}_{11}^{4}$ & 5.598338 \\
12 & $\mathrm{~A}_{12}^{*}$ & 7.510113 \\
13 & $\mathrm{~A}_{13}^{7}$ & 7.864060 \\
14 & $\mathrm{~A}_{14}^{5}$ & 9.006610 \\
15 & $\mathrm{~A}_{15}^{8}$ & 11.601626 \\
16 & $\mathrm{~A}_{16}^{*}$ & 15.310927 \\
17 & $\mathrm{~A}_{17}^{*}$ & 18.287811 \\
18 & $\mathrm{~A}_{18}^{*}$ & 21.840949 \\
19 & $\mathrm{~A}_{19}^{*}$ & 26.081820 \\
20 & $\mathrm{~A}_{20}^{*}$ & 31.143448 \\
21 & $\mathrm{~A}_{21}^{*}$ & 37.184568 \\
22 & $\Lambda_{22}^{*}$ & $\leq 27.8839$ \\
23 & $\Lambda_{23}^{*}$ & $\leq 15.3218$ \\
24 & $\Lambda_{24}$ & 7.903536 \\
\hline & &
\end{tabular}

coverings. Their covering densities were estimated by Smith [59], but we do not know the exact values of $\Theta\left(\Lambda_{22}^{*}\right)$ and $\Theta\left(\Lambda_{23}^{*}\right)$. For the definitions of the root lattices $A_{n}, D_{n}, E_{n}$, and the laminated lattices $\Lambda_{n}$, we refer to Chapters 4 and 6 of [22].

In [24] Coxeter gave a list of locally optimal lattice packings (extreme lattices) which are related to Lie groups. One of them is the infinite series of locally densest packing lattices $\mathrm{A}_{d}^{r}$ where $d \geq 2$ and $r>1$ divides $d+1$. The lattice $\mathrm{A}_{d}^{r}$ is the unique sublattice of $\mathrm{A}_{d}^{*}$ containing $\mathrm{A}_{d}$ to index $r$. In [10] Baranovskii determined the covering density of the lattice covering given by $A_{9}^{5}$, which is slightly better than the one given by $A_{9}^{*}$. Recently, Anzin extended Baranovskii's work. In [2] he computed the covering densities of $A_{11}^{4}$ and $A_{13}^{7}$, and in a private communication he reported on computing the covering densities of $A_{14}^{5}$ and $A_{15}^{8}$. They all give less dense lattice coverings than those provided by the corresponding $A_{d}^{*}$. We do not know whether these lattice coverings are locally optimal.

To answer Ryshkov's question exhaustively it will be necessary to investigate lattice coverings in the dimensions $d=10,12,16,17, \ldots, 21$. We hope that the methods we present in this paper will be useful for this project.

\section{The Lattice Packing-Covering Problem}

The lattice packing-covering problem has been studied in different contexts and there are several different names and interpretations of the lattice packing-covering constant $\gamma_{d}$. Lagarias and Pleasants [46] referred to it as the "Delone packing-covering constant". 


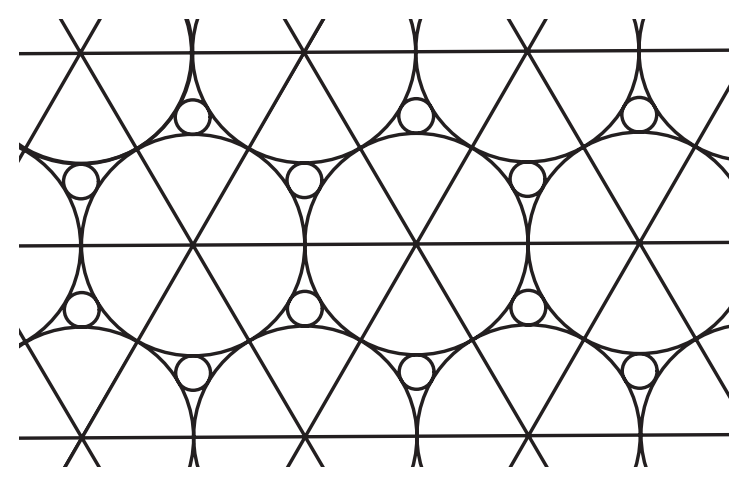

Fig. 2. A close sphere packing given by the hexagonal lattice.

Ryshkov [52] studied the equivalent problem of minimizing the density of $(r, R)$-systems. An $(r, R)$-system is a discrete point set $X \subseteq \mathbb{R}^{d}$ where (1) the distance between any two points of $X$ is at least $r$ and (2) the distance from any point in $\mathbb{R}^{d}$ to a point in $X$ is at most $R$. If $X$ is a lattice, then $r=\lambda(X) / 2$ and $R=\mu(X)$.

Geometrically we may think of solving the lattice packing-covering problem as maximizing the minimum distance between lattice points in a lattice covering with unit spheres. Alternatively, we may think of it as minimizing the radius of a largest sphere that could additionally be packed into a lattice packing of unit spheres (see Fig. 2). This minimal gap-radius is equal to $\gamma_{d}-1$. Therefore the problem raised by L. Fejes Tóth [36] of finding "close packings" attaining this gap-radius is another formulation of the packing-covering problem.

The last interpretation shows that $\gamma_{d} \geq 2$ would imply that in any $d$-dimensional lattice packing with spheres of unit radius there is still space for spheres of radius 1 . In particular, this would prove that densest sphere packings in dimension $d$ are non-lattice packings. This phenomenon is likely to be true for large dimensions, but it has not been verified for any $d$ so far.

Problem 4.1. Does there exist a $d$ such that $\gamma_{d} \geq 2$ ?

Note that this problem is particularly challenging in view of the asymptotic bound $\gamma_{d} \leq 2+o(1)$ due to Butler [21].

As for the lattice covering problem, the lattice packing-covering problem has been solved up to dimension 5 (see Table 3). Ryshkov [52] solved the general two-dimensional case. The three-dimensional case was settled by Böröczky [19], even without the restriction to lattices. The four- and five-dimensional cases were solved by Horváth [43], [44]. Note that the lattices $\mathrm{Ho}_{4}$ and $\mathrm{Ho}_{5}$ (see Section 11) discovered by Horváth are neither best covering nor best packing lattices. As in the case of lattice coverings, the results were attained by using Voronoi's reduction theory.

Our computations, described in Section 11, verify all of the known results in dimension $\leq 5$. As for the covering problem, none of the values $\gamma_{d}$ has been determined in a dimension $d \geq 6$ so far. In Section 11 we report on a new best known packing-covering lattice for $d=6$. We thereby show in particular that $\gamma_{6}<1.412$, revealing the phenomenon 
Table 3. Optimal packing-covering lattices.

\begin{tabular}{|c|c|c|}
\hline$d$ & Lattice & Lattice packing-covering constant $\gamma_{d}$ \\
\hline 1 & $\mathbb{Z}^{1}$ & 1 \\
\hline 2 & $A_{2}^{*}$ & $2 / \sqrt{3} \approx 1.154700$ \\
\hline 3 & $A_{3}^{*}$ & $\sqrt{5 / 3} \approx 1.290994$ \\
\hline 4 & $\mathrm{Ho}_{4}$ & $\sqrt{2 \sqrt{3}}(\sqrt{3}-1) \approx 1.362500$ \\
\hline 5 & $\mathrm{Ho}_{5}$ & $\sqrt{3 / 2+\sqrt{13} / 6} \approx 1.449456$ \\
\hline
\end{tabular}

$\gamma_{6}<\gamma_{5}$, recently suspected by Lagarias and Pleasants [46, Section 7]. Note that this was already observed by Zong [66, Remark 3], who showed that $\gamma\left(E_{6}^{*}\right)=\sqrt{2}<\gamma_{5}$.

We were not able yet to find any new best known lattices in dimensions $d \geq 7$. The lattice $\mathrm{E}_{7}^{*}$ gives the best known lattice in dimension 7. Nevertheless, because of their symmetry and the known bounds on $\gamma_{d}$, Zong [66, Conjecture 3.1] made the following conjectures: $E_{8}$ and Leech lattice $\Lambda_{24}$ are optimal in their dimensions. In [57] we showed that the Leech lattice gives a locally optimal lattice packing-covering constant. It is an open question whether or not the root lattice $\mathrm{E}_{8}$ gives a locally optimal lattice packingcovering constant as well. The corresponding values of the lattice packing-covering constant are shown in Table 4. In dimension 7 and between dimensions 8 and 24 we do not yet know enough to state any serious conjectures. The exact value for the smallest known lattice packing-covering constant in dimension 6 is $2 \sqrt{2 \sqrt{798}-56}$ (see Section 11).

\section{Voronoi's Reduction Theory}

The general task of a reduction theory for $\mathrm{PQFs}$ is to give a fundamental domain for $\mathcal{S}_{>0}^{d} / \mathrm{GL}_{d}(\mathbb{Z})$. This is a subset which behaves like $\mathcal{S}_{>0}^{d} / \mathrm{GL}_{d}(\mathbb{Z})$ up to boundary identifications. There are many different reduction theories, connected with names like Lagrange, Gauß, Hermite, Korkine, Zolotareff, Minkowski, Voronoi, and others (see [55]). In this section we describe the reduction theory developed by Voronoi in [64]. It is based on Delone triangulations.

Table 4. Lattice packing-covering records.

\begin{tabular}{rcr}
\hline$d$ & Lattice & Lattice packing-covering constant $\gamma$ \\
\hline 6 & $\mathrm{~L}_{6}^{p c}$ & $2 \sqrt{2 \sqrt{798}-56} \approx 1.411081$ \\
7 & $\mathrm{E}_{7}^{*}$ & $\sqrt{7 / 3} \approx 1.527525$ \\
8 & $\mathrm{E}_{8}$ & $\sqrt{2} \approx 1.414213$ \\
24 & $\Lambda_{24}$ & $\sqrt{2} \approx 1.414213$ \\
\hline
\end{tabular}




\subsection{Secondary Cones of Delone Triangulations}

Let $Q \in \mathcal{S}_{>0}^{d}$ be a PQF. A polytope $L=\operatorname{conv}\left\{\boldsymbol{v}_{1}, \ldots, \boldsymbol{v}_{n}\right\}$, with $\boldsymbol{v}_{1}, \ldots, \boldsymbol{v}_{n}$ in $\mathbb{Z}^{d}$, is called a Delone polytope of $Q$ if there exists a $c \in \mathbb{R}^{d}$ and a real number $r \in \mathbb{R}$ with $Q\left[\boldsymbol{v}_{i}-\boldsymbol{c}\right]=r^{2}$ for all $i=1, \ldots, n$, and for all other lattice points $\boldsymbol{v} \in \mathbb{Z}^{d} \backslash\left\{\boldsymbol{v}_{1}, \ldots, \boldsymbol{v}_{n}\right\}$ we have strict inequality $Q[\boldsymbol{v}-\boldsymbol{c}]>r^{2}$. The set of all Delone polytopes

$$
\operatorname{Del}(Q)=\{L: L \text { is a Delone polytope of } Q\}
$$

is called the Delone subdivision (or L-partition) of $Q$. A Delone triangulation is a Delone subdivision that consists of simplices only. For historical reasons we use the letter $L$ to denote Delone polytopes (see [64] and [26]).

The Delone subdivision of a PQF is a periodic polytopal subdivision of $\mathbb{R}^{d}$. We say that two Delone polytopes $L$ and $L^{\prime}$ are equivalent if there is a $v \in \mathbb{Z}^{d}$ with $L^{\prime}=L+\boldsymbol{v}$. Given a Delone subdivision $\mathcal{D}$ of $\mathbb{R}^{d}$, the set of PQFs with Delone subdivision $\mathcal{D}$ forms the secondary cone

$$
\Delta(\mathcal{D})=\left\{Q \in \mathcal{S}_{>0}^{d}: \operatorname{Del}(Q)=\mathcal{D}\right\}
$$

In the literature it is often referred to as the L-type domain of $Q \in \Delta(\mathcal{D})$. We prefer the term "secondary cone" because of the close connection of Voronoi's reduction theory to the theory of secondary polytopes which we point out in Section 5.5.

Let $Q$ be a PQF whose Delone subdivision is a triangulation of $\mathbb{R}^{d}$. In the following we describe the secondary cone of $\operatorname{Del}(Q)$. For this, let $L=\operatorname{conv}\left\{\boldsymbol{v}_{1}, \ldots, \boldsymbol{v}_{d+1}\right\}$ and $L^{\prime}=\operatorname{conv}\left\{\boldsymbol{v}_{2}, \ldots, \boldsymbol{v}_{d+2}\right\}$ be two $d$-dimensional Delone simplices of $Q$ sharing the common facet $F=\operatorname{conv}\left\{\boldsymbol{v}_{2}, \ldots, \boldsymbol{v}_{d+1}\right\}$. Let $\alpha_{1}, \ldots, \alpha_{d+2}$ be real numbers with $\alpha_{1}=1$, $\sum_{i=1}^{d+2} \alpha_{i}=0$ and $\sum_{i=1}^{d+2} \alpha_{i} \boldsymbol{v}_{i}=\boldsymbol{O}$ (hence $\alpha_{d+2}>0$ ). The regulator of the pair of adjacent simplices $\left(L, L^{\prime}\right)$ is the linear form $\varrho_{\left(L, L^{\prime}\right)}\left(Q^{\prime}\right)=\sum_{i=1}^{d+2} \alpha_{i} Q^{\prime}\left[\boldsymbol{v}_{i}\right], Q^{\prime} \in \mathcal{S}^{d}$. In particular, note that the regulator solely depends on the points $v_{1}, \ldots, v_{d+2}$, that $\varrho_{\left(L, L^{\prime}\right)}(Q)>0$ and that $\varrho_{\left(L, L^{\prime}\right)}=\varrho_{\left(L+v, L^{\prime}+v\right)}$ for all $\boldsymbol{v} \in \mathbb{Z}^{d}$. One can describe $\boldsymbol{\Delta}(\operatorname{Del}(Q))$ by linear inequalities coming from the (finitely many) regulators of $\operatorname{Del}(Q)$ :

Proposition 5.1 [64, Section 77]. Let $Q$ be a PQF whose Delone subdivision is a triangulation. The secondary cone of the Delone triangulation $\operatorname{Del}(Q)$ is the full-dimensional open polyhedral cone

$$
\begin{aligned}
\Delta(\operatorname{Del}(Q))= & \left\{Q^{\prime} \in \mathcal{S}^{d}: \varrho_{\left(L, L^{\prime}\right)}\left(Q^{\prime}\right)>0, \text { for all pairs }\left(L, L^{\prime}\right)\right. \\
& \text { of adjacent simplices of } \operatorname{Del}(Q)\} .
\end{aligned}
$$

Note on the one side that $\Delta(\operatorname{Del}(Q))$ is contained in $\mathcal{S}_{>0}^{d}$ by definition and on the other side that the linear inequalities given by the regulators imply that a quadratic form which satisfies them is positive definite.

Example 5.2. As a first example and because of its importance for the lattice problems introduced in Section 2, we describe the Delone subdivision of Voronoi's principal form of the first type $Q[x]=d \sum x_{i}^{2}-\sum x_{i} x_{j}$, which is associated to the lattice $\mathrm{A}_{d}^{*}$, in greater detail. The Delone subdivision of $Q$ is a triangulation and can be described as follows: 


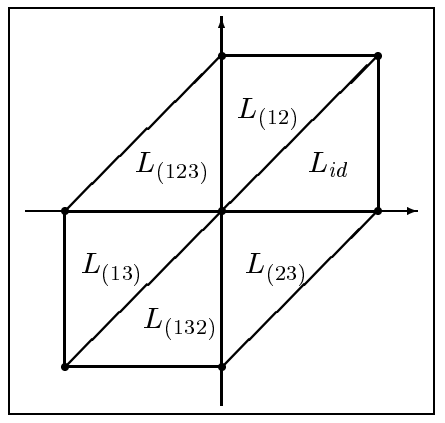

Fig. 3. The triangulation $\mathcal{D}_{1}$ in dimension $d=2$.

Let $\boldsymbol{e}_{1}, \ldots, \boldsymbol{e}_{d}$ be the standard basis vectors of $\mathbb{Z}^{d}$, and set $\boldsymbol{e}_{d+1}=-\boldsymbol{e}_{1}-\cdots-\boldsymbol{e}_{d}$. For a permutation $\pi \in \mathrm{S}_{d+1}$ we define the $d$-dimensional simplex $L_{\pi}$ by

$$
L_{\pi}=\operatorname{conv}\left\{\boldsymbol{e}_{\pi(1)}, \boldsymbol{e}_{\pi(1)}+\boldsymbol{e}_{\pi(2)}, \ldots, \boldsymbol{e}_{\pi(1)}+\cdots+\boldsymbol{e}_{\pi(d+1)}\right\} .
$$

The set of simplices $\left\{L_{\pi}+v: v \in \mathbb{Z}^{d}, \pi \in \mathrm{S}_{d+1}\right\}$ defines a triangulation of $\mathbb{R}^{d}$ which we from now on denote by $\mathcal{D}_{1}$. The full-dimensional cells containing the origin are $L_{\pi}$, $\pi \in \mathrm{S}_{d+1}$. Two simplices $L_{\pi}$ and $L_{\pi^{\prime}}$ have a facet in common if and only if $\pi$ and $\pi^{\prime}$ differ by a single transposition of two adjacent positions. The automorphism group of $\mathcal{D}_{1}$ is isomorphic to the permutation group $\mathrm{S}_{d+1}$. The star of the origin is illustrated in Fig. 3. This consists of all Delone polytopes containing the origin.

The secondary cone of $\mathcal{D}_{1}$ is (see Sections 102-104 of [64])

$$
\Delta\left(\mathcal{D}_{1}\right)=\left\{Q \in \mathcal{S}^{d}: q_{i j}<0 \text { for } i \neq j \text { and } \sum_{i} q_{i j}>0 \text { for } j=1, \ldots, d\right\} .
$$

Its topological closure $\overline{\boldsymbol{\Delta}\left(\mathcal{D}_{1}\right)}$ is called Voronoi's principal domain of the first type.

It was shown by Voronoi [64, Section 97] that the topological closures of the secondary cones gives a facet-to-facet tessellation of $\mathcal{S}_{\geq 0}^{d}$. By a theorem of Gruber and Ryshkov [42] we even have a face-to-face tessellation because "facet-to-facet implies face-toface". This means that a face sharing relative interior points with another face of its dimension coincides with this face, whenever this property holds for the facets (faces of co-dimension 1).

\subsection{Bistellar Operations}

Now, given a secondary cone, how do we find its neighbors, that is, those secondary cones sharing a facet with the given one?

An answer can be given by taking a closer look at the so-called repartitioning polytopes (introduced by Ryshkov and Baranovskii in [54]), which are "hidden" in the definition of the regulators. Repartitioning polytopes are $d$-dimensional Delone polytopes having 
a representation as the convex hull of two Delone simplices sharing a common facet. Thus, repartitioning polytopes have $d+2$ vertices.

Generally, $d$-dimensional polytopes with $d+2$ vertices have the special property that there are exactly two different ways to triangulate them: Let $V$ be a set of $d+2$ points which affinely spans $\mathbb{R}^{d}$. Let $\sum_{\boldsymbol{v} \in V} \alpha_{\boldsymbol{v}} \boldsymbol{v}=\boldsymbol{0}, \sum_{\boldsymbol{v} \in V} \alpha_{\boldsymbol{v}}=0$, be an affine relation between these points. There exist exactly two triangulations of conv $V: \mathcal{T}_{+}(V, \boldsymbol{\alpha})$ with $d$-simplices $\operatorname{conv}(V \backslash\{\boldsymbol{v}\}), \alpha_{v}>0$, and $\mathcal{T}_{-}(V, \boldsymbol{\alpha})$ with $d$-simplices $\operatorname{conv}(V \backslash\{\boldsymbol{v}\}), \alpha_{v}<0$ (see for example Proposition 1.2 in Chapter 7 of [40]).

Let $\mathcal{D}$ be a Delone triangulation of $\mathbb{R}^{d}$ and let $F$ be a $(d-1)$-dimensional cell of $\mathcal{D}$. Then $F$ is contained in two simplices $L$ and $L^{\prime}$ of $\mathcal{D}$. By $V$ we denote the set of vertices of $L$ and $L^{\prime}, V=\operatorname{vert} L \cup$ vert $L^{\prime}$. By $\alpha$ we denote an affine relation between the points in $V$. The $(d-1)$-dimensional cell $F$ is called a flippable facet of the triangulation $\mathcal{D}$ if one of the triangulations $\mathcal{T}_{+}(V, \boldsymbol{\alpha})$ or $\mathcal{T}_{-}(V, \boldsymbol{\alpha})$ is a subcomplex of $\mathcal{D}$. If $F$ is a flippable facet of $\mathcal{D}$ and we replace the subcomplex $\mathcal{T}_{+}(V, \boldsymbol{\alpha})$ by $\mathcal{T}_{-}(V, \boldsymbol{\alpha})$ (respectively $\mathcal{T}_{-}(V, \boldsymbol{\alpha})$ by $\mathcal{I}_{+}(V, \boldsymbol{\alpha})$ ), then we get a new triangulation. This replacement is called a bistellar operation or flip.

Notice that non-flippable facets do exist and that performing a bistellar operation in a Delone triangulation does not necessarily produce a Delone triangulation. Both phenomena occur starting from dimension 4.

Nevertheless, the facets of $\overline{\boldsymbol{\Delta}(\mathcal{D})}$ correspond exactly to those bistellar operations of $\mathcal{D}$ which yield new Delone triangulations. A $(d-1)$-dimensional cell $L \cap L^{\prime} \in \mathcal{D}$ is a flippable facet whenever the corresponding regulator $\varrho_{\left(L, L^{\prime}\right)}$ gives a facet-defining hyperplane of $\overline{\Delta(\mathcal{D})}$ (see Sections 87 and 88 of [64]). This is clear since the repartitioning polytope $\operatorname{conv}(L \cup L)$ is a Delone polytope of the PQFs lying in the relative interior of the facet given by $\varrho_{\left(L, L^{\prime}\right)}$.

Let $\mathbf{F}$ be a facet of $\overline{\Delta(\mathcal{D})}$. We describe how the Delone triangulation $\mathcal{D}$ changes if we vary a PQF continuously: We start from the interior of $\overline{\Delta(\mathcal{D})}$, then we move towards a relative interior point of $\mathbf{F}$ and finally we go infinitesimally further, leaving $\overline{\Delta(\mathcal{D})}$. In every repartitioning polytope $V=\operatorname{conv}\left(L \cup L^{\prime}\right)$ where $L, L^{\prime}$ is a pair of adjacent simplices whose regulator defines $\mathbf{F}$, i.e. the linear span of $F$ satisfies $\operatorname{lin} \mathbf{F}=\{Q \in$ $\left.\mathcal{S}^{d}: \varrho_{\left(L, L^{\prime}\right)}(Q)=0\right\}$, we perform a bistellar operation. This gives a new triangulation $\mathcal{D}^{\prime}$, which is again a Delone triangulation. The two secondary cones $\overline{\Delta(\mathcal{D})}$ and $\overline{\Delta\left(\mathcal{D}^{\prime}\right)}$ have the complete facet $\mathbf{F}$ in common. We say that $\mathcal{D}$ and $\mathcal{D}^{\prime}$ are bistellar neighbors. In Sections 91-96 of [64], Voronoi computes the secondary cone of $\mathcal{D}^{\prime}$ explicitly and shows that $\overline{\boldsymbol{\Delta}\left(\mathcal{D}^{\prime}\right)}$ has dimension $d(d+1) / 2$.

\subsection{Main Theorem of Voronoi's Reduction Theory}

By constructing bistellar neighbors we could produce infinitely many Delone triangulations starting from the Delone triangulation $\mathcal{D}_{1}$ of Voronoi's principal form of the first type (a part of the infinite flip graph of two-dimensional Delone triangulations is given in Fig. 4). Many of these will not be essentially new, because the group $\mathrm{GL}_{d}(\mathbb{Z})$ is acting on the set of Delone subdivisions by $(A, \mathcal{D}) \mapsto A \mathcal{D}$ and it is acting on the set of secondary cones by $(A, \boldsymbol{\Delta}) \mapsto A^{t} \Delta A$ for $A \in \mathrm{GL}_{d}(\mathbb{Z})$. We are only interested in the orbits of these 


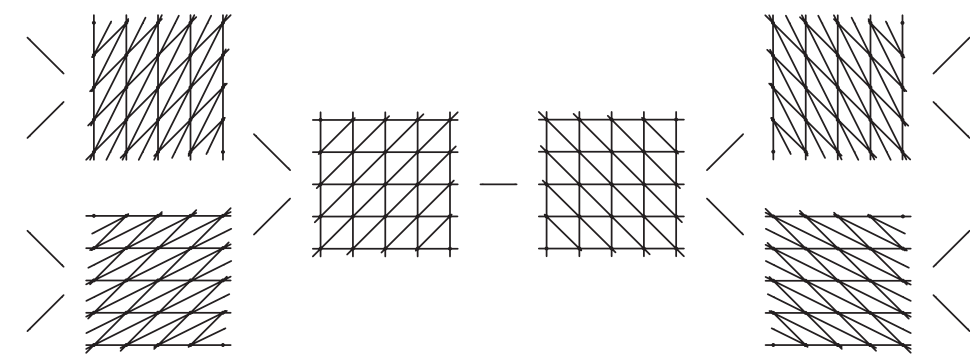

Fig. 4. The graph of two-dimensional Delone triangulations.

group actions and there are only finitely many, as shown by Voronoi [64, Section 98] (see also Chapter 13.3 of [30]). Altogether we get:

Theorem 5.3 (Main Theorem of Voronoi's Reduction Theory). The secondary cone of a Delone triangulation is a full-dimensional, open polyhedral cone in $\mathcal{S}_{\geq 0}^{d}$. The topological closures of secondary cones of Delone triangulations give a face-to-face tiling in $\mathcal{S}_{\geq 0}^{d}$. Two secondary cones share a facet if and only if they are bistellar neighbors. The group $\mathrm{GL}_{d}(\mathbb{Z})$ acts on the tiling, and under this group action there are only finitely many inequivalent secondary cones.

The main theorem translates into Algorithm 1 which enumerates all inequivalent Delone triangulations in a given dimension. We developed the program scc (secondary cone cruiser) which is an implementation of Algorithm 1. The interested reader can download SCC from our web page [58]. Using our implementation we succeeded in reproducing the classification of all inequivalent Delone triangulations up to dimension 5. Table 5 shows the numbers.

Input: Dimension $d$.

Output: Set $\mathcal{R}$ of all inequivalent $d$-dimensional Delone triangulations.

$T \leftarrow\left\{\mathcal{D}_{1}\right\}$, where $\mathcal{D}_{1}$ is the Delone triangulation described in Example 5.2.

$\mathcal{R} \leftarrow \emptyset$.

while there is a $\mathcal{D} \in T$ do

$T \leftarrow T \backslash\{\mathcal{D}\} . \mathcal{R} \leftarrow \mathcal{R} \cup\{\mathcal{D}\}$.

compute the regulators of $\mathcal{D}$.

compute the facets $F_{1}, \ldots, F_{n}$ of $\overline{\Delta(\mathcal{D})}$.

for $i=1, \ldots, n$ do

compute the bistellar neighbor $\mathcal{D}_{i}$ of $\mathcal{D}$ which is defined by $F_{i}$.

if $\mathcal{D}_{i}$ is not equivalent to a Delone triangulation in $\mathcal{R} \cup\left\{\mathcal{D}_{1}, \ldots, \mathcal{D}_{i-1}\right\}$ then

$$
T \leftarrow T \cup\left\{\mathcal{D}_{i}\right\}
$$

end if

end for

end while

Algorithm 1. Enumeration of all inequivalent Delone triangulations. 
Table 5. Numbers of inequivalent Delone triangulations.

\begin{tabular}{lllllc}
\hline Dimension & 1 & 2 & 3 & 4 & 5 \\
\# Delone triangulations & 1 & 1 & 1 & 3 & 222 \\
\hline
\end{tabular}

We are not the first to compute this classification. Voronoi performed the classification of all inequivalent Delone triangulation up to dimension 4 in his memoir [64]. In [11] and [54] Ryshkov and Baranovskii reported on 221 inequivalent Delone triangulation in dimension 5. However, they missed one type which was found by Engel [33]. Engel and Grishukhin [35] undertook the non-trivial task of identifying the Delone triangulation missing in the list of Ryshkov and Baranovskii. There they also report on several errors in both lists. Our computations confirm that the number of Delone triangulations in dimension 5 is 222. Beginning with dimension 6 the number of inequivalent Delone triangulations starts to explode. We found more than 250, 000 inequivalent triangulations before our implementation stopped because of memory reasons. We do not know how many inequivalent triangulations we have to expect in dimension 6 but we are quite certain that we only saw a small fraction of them. No non-trivial bounds on the number of inequivalent Delone triangulations for general dimension $d$ are known.

\subsection{Degeneracy}

Until now we have only dealt with Delone triangulations of positive definite quadratic forms. Let us look at possible degenerations-Delone subdivisions of positive semidefinite quadratic forms - and find out how they fit into the theory developed so far.

Let $Q$ be a positive semidefinite quadratic form which is arithmetically equivalent to $\left(\begin{array}{ll}0 & 0 \\ 0 & Q^{\prime}\end{array}\right)$ where $Q^{\prime}$ is positive definite. Then we can use the definition of Delone subdivision almost literally; we only have to replace "polytope" by "polyhedron" (a polyhedron is the intersection of finitely many half-spaces; a polytope is a bounded polyhedron).

Delone subdivisions are limiting cases of triangulations. Their secondary cones occur on the boundaries of full-dimensional secondary cones of Delone triangulations. Let $\mathcal{D}$ and $\mathcal{D}^{\prime}$ be two Delone subdivisions. We say $\mathcal{D}$ is a refinement of $\mathcal{D}^{\prime}$ if every Delone polytope of $\mathcal{D}$ is a subset of some Delone polytope of $\mathcal{D}^{\prime}$. The following proposition, which seems to be folklore, shows that the relation between refinements, secondary cones and sums of positive semidefinite quadratic forms is very natural. One can find a proof for example in [62, Proposition 2.6.1].

Proposition 5.4. Let $\mathcal{D}$ be a Delone triangulation.

1. A positive semidefinite quadratic form $Q$ lies in $\overline{\boldsymbol{\Delta}(\mathcal{D})}$ if and only if $\mathcal{D}$ is a refinement of $\operatorname{Del}(Q)$.

2. If two positive semidefinite quadratic forms $Q$ and $Q^{\prime}$ both lie in $\overline{\Delta(\mathcal{D})}$, then $\operatorname{Del}\left(Q+Q^{\prime}\right)$ is a common refinement of $\operatorname{Del}(Q)$ and $\operatorname{Del}\left(Q^{\prime}\right)$.

Therefore, the classification of all inequivalent Delone subdivisions is equivalent to the classification of all inequivalent secondary cones. This has been done up to dimension 5 . 
Table 6. Numbers of inequivalent Delone subdivisions.

\begin{tabular}{llllcc}
\hline Dimension & 1 & 2 & 3 & 4 & 5 \\
\# Delone subdivisions & 1 & 2 & 5 & 52 & 179,372 \\
\hline
\end{tabular}

The one- and two-dimensional cases are trivial; the three-dimensional case goes back to Federov, who classified all polytopes which tile three-dimensional space by translates in 1885. Delone [25] (later corrected by Stogrin [60]) found 51 of the 52 Delone subdivisions in dimension 4. Recently, Engel [34] reported that there are 179,372 inequivalent five-dimensional Delone subdivisions. It is possible to verify his result by enumerating the faces of the 222 secondary cones of Delone triangulations in dimension 5 up to equivalence. Since we do not need Engel's result for our application we did not verify it. Again, the number in dimension 6 is not known and will be much larger. Table 6 summarizes this discussion.

\subsection{Generalized Secondary Polytopes}

Triangulations of discrete point sets have attracted many researchers in recent years. They have many applications, for example in computational geometry, optimization, algebraic geometry, topology, etc. One main tool to understand the structural behavior of triangulations of finite point sets is the theory of secondary polytopes invented by Gel'fand et al. [40, Chapter 7].

Let $\mathcal{A}=\left\{\boldsymbol{a}_{1}, \ldots, \boldsymbol{a}_{n}\right\} \subseteq \mathbb{R}^{d}$ be a finite set of points. Let $w: \mathcal{A} \rightarrow \mathbb{R}$ be a map that assigns to every point in $\mathcal{A}$ a weight. The set of weight maps forms a vector space over $\mathbb{R}$ which we denote by $\mathbb{R}^{\mathcal{A}}$. A lifting map $l: \mathcal{A} \rightarrow \mathbb{R}^{d} \times \mathbb{R}, l\left(\boldsymbol{a}_{i}\right)=\left(\boldsymbol{a}_{i}, w\left(\boldsymbol{a}_{i}\right)\right)$ is defined by $w$ which lifts each point $\boldsymbol{a}_{i} \in \mathcal{A}$ by its weight $w\left(\boldsymbol{a}_{i}\right)$. A subdivision of the convex polytope conv $\mathcal{A}$ is induced by $l$ : We take the convex hull of the lifted points $\operatorname{conv} l(\mathcal{A})$ and project its lower faces as seen from $(\boldsymbol{0},-\infty)$ back down onto $\mathbb{R}^{d}$. A subdivision that can be obtained in this manner is called a regular subdivision. Delone subdivisions (or more precisely Delone subdivisions of finitely many points) are regular subdivisions since the underlying positive semidefinite quadratic form is the weight function. This view on Delone subdivisions was introduced by Brown [20] and by Edelsbrunner and Seidel [32].

Let $\mathcal{T}$ be a regular triangulation of conv $\mathcal{A}$. We may ask what are the weight functions which define $\mathcal{T}$. What is the secondary cone of $\mathcal{T}$ in the parameter space $\mathbb{R}^{\mathcal{A}}$ ? As in Voronoi's reduction theory it turns out that the secondary cone of $\mathcal{T}$ is a full-dimensional open polyhedral cone. The topological closures of the secondary cones of all regular triangulations tile the space $\mathbb{R}^{\mathcal{A}}$ face-to-face. The tiling is called the secondary fan of $\mathcal{A}$. If two secondary cones have a facet in common, then the corresponding regular triangulations differ by a bistellar operation in exactly one "repartitioning polytope" (in this context it is a polytope with $d+2$ vertices without the condition of being a Delone polytope) that is defined by the facet. The faces in the secondary fan $\mathcal{A}$ are in a one-toone correspondence to regular subdivisions in essentially the same way we discussed in Section 5.4 for Delone subdivisions. 
So far we have seen that the theory of regular subdivisions of finite point sets and the theory of Delone subdivisions of the lattice $\mathbb{Z}^{d}$ can be developed analogously, but there are also differences: The parameter space for regular subdivisions is the vector space $\mathbb{R}^{\mathcal{A}}$, while for Delone subdivisions it is the pointed cone $\mathcal{S}_{\geq 0}^{d}$. Groups play an important role for Delone subdivisions. The group $\mathbb{Z}^{d}$ is acting on Delone subdivisions by translations. The group $\mathrm{GL}_{d}(\mathbb{Z})$ is acting on the set of secondary cones.

If we order all regular subdivisions of conv $\mathcal{A}$ by refinement we get a poset. This poset has a very nice combinatorially structure as proved by Gel'fand et al.: There exists a polytope-the secondary polytope $\Sigma(\mathcal{A})$ of $\mathcal{A}$-whose normal fan equals the secondary fan of $\mathcal{A}$. So the refinement poset is anti-isomorphic to the face lattice of the secondary polytope. Regular triangulations are in one-to-one correspondence to the vertices, two regular triangulations differ by a bistellar operation if and only if their vertices are connected by an edge, etc.

Recently, a similar combinatorial structure lurking behind the refinement poset of $\mathbb{Z}^{d}$-periodic subdivisions (this poset contains the poset of Delone subdivisions) has been described by Alexeev [1, Sections 5.11 and 5.12]. He gives an unbalanced and a balanced version of these generalized secondary polytopes, where the latter one is invariant with respect to the group action of $\mathrm{GL}_{d}(\mathbb{Z})$.

\section{Convex Optimization with LMI Constraints}

In this section we introduce determinant maximization problems, which are convex programming problems with linear matrix inequality (LMI) constraints. In a sense they are equivalent to the better known semidefinite programming problems. Efficient algorithms and implementations are available for both classes. In Section 8 we see how Problems 2.1 and 2.2 can be naturally formulated as a finite number of determinant maximization problems.

Following Vandenberghe et al. [63] we say that a determinant maximization problem is an optimization problem of the form

$$
\begin{array}{ll}
\text { minimize } & \boldsymbol{c}^{t} \boldsymbol{x}-\log \operatorname{det} G(\boldsymbol{x}) \\
\text { subject to } & G(\boldsymbol{x}) \text { is a positive definite matrix } \\
& F(\boldsymbol{x}) \text { is a positive semidefinite matrix. }
\end{array}
$$

The optimization vector is $\boldsymbol{x} \in \mathbb{R}^{d}$, the objective vector is $c \in \mathbb{R}^{d}$ and $G: \mathbb{R}^{d} \rightarrow \mathbb{R}^{m \times m}$ and $F: \mathbb{R}^{d} \rightarrow \mathbb{R}^{n \times n}$ are affine maps:

$$
\begin{aligned}
& G(\boldsymbol{x})=G_{0}+x_{1} G_{1}+\cdots+x_{d} G_{d}, \\
& F(\boldsymbol{x})=F_{0}+x_{1} F_{1}+\cdots+x_{d} F_{d},
\end{aligned}
$$

where $G_{i} \in \mathbb{R}^{m \times m}$ and $F_{i} \in \mathbb{R}^{n \times n}$, for $i=0, \ldots, d$, are symmetric matrices. In what follows we write $G(\boldsymbol{x}) \succ 0$ and $F(\boldsymbol{x}) \succeq 0$ for the LMIs defining the constraints of the determinant maximization problem. As a special case, our formulation reduces to a semidefinite programming problem whenever $G(\boldsymbol{x})$ is the identity matrix for all $\boldsymbol{x} \in \mathbb{R}^{d}$.

Currently there exist two different types of algorithms-ellipsoid and interior-point methods-which efficiently solve semidefinite programming problems. They can ap- 
proximate the solution of a semidefinite programming problem within any specified accuracy and run in polynomial time if the instances are "well-behaved". For more information on the exciting topic of semidefinite programming the interested reader is referred to the vast amount of literature which to a great extent is available on the World Wide Web. A good starting point is the web page ${ }^{1}$ of Christoph Helmberg.

Nesterov and Nemirovskii [48] developed a framework for the design of efficient interior-point algorithms for general and specific classes of convex programming problems. They also showed (Section 6.4.3) that the determinant maximization problem can be transformed into a semidefinite programming problem by a transformation which can be computed in polynomial time. Nevertheless it is faster to solve the determinant maximization problem directly. Vandenberghe et al. [63] and independently Toh [61] gave interior-point algorithms for the determinant maximization problem. Both algorithms fit into the general framework of Nesterov and Nemirovskii. For our implementation we use the software package MAXDET ${ }^{2}$ of $\mathrm{Wu}$, Vandenberghe, and Boyd as a subroutine.

One nice feature of determinant maximization problems is that there is a duality theory similar to the one of linear programming, which allows one to compute certificates for a range in which the optimum is attained, the so-called duality gap. The dual problem of problem (1) is (see Section 3 of [63])

\begin{tabular}{|ll|}
\hline maximize & $\log \operatorname{det} W-\operatorname{trace}\left(G_{0} W\right)-\operatorname{trace}\left(F_{0} Z\right)+m$ \\
subject to & $\operatorname{trace}\left(G_{i} W\right)+\operatorname{trace}\left(F_{i} Z\right)=\boldsymbol{c}_{i}$ for $i=1, \ldots, d$, \\
& $W \succ 0, Z \succeq 0$. \\
\hline
\end{tabular}

Here, $W \in \mathbb{R}^{m \times m}$ and $Z \in \mathbb{R}^{n \times n}$ are symmetric matrices of the same size as the $G_{i}$ and $F_{i}$ respectively.

The knowledge of a vector $\boldsymbol{x}$ with $G(\boldsymbol{x}) \succ 0$ and $F(\boldsymbol{x}) \succeq 0$, and of a pair $(W, Z)$, with $\operatorname{trace}\left(G_{i} W\right)+\operatorname{trace}\left(F_{i} Z\right)=\boldsymbol{c}_{i}$ for $i=1, \ldots, d$, and $W \succ 0$, and $Z \succeq 0$, gives upper and lower bounds for the optimal value $p^{*}$ of (1) by

$$
\log \operatorname{det} W-\operatorname{trace}\left(G_{0} W\right)-\operatorname{trace}\left(F_{0} Z\right)+m \leq p^{*} \leq \boldsymbol{c}^{t} \boldsymbol{x}-\log \operatorname{det} G(\boldsymbol{x}) .
$$

If $\boldsymbol{x}, W$ and $Z$ have rational entries only, we can compute lower and upper bounds of $p^{*}$ that are mathematical rigorous. For the proofs in Section 11 we have therefore developed a program rma (rigorous MAXDET) which calls MAXDET to find floating-point values of a vector $\boldsymbol{x}$ and a pair $(W, Z)$ satisfying the constraints. Then it approximates $\boldsymbol{x}$ and $(W, Z)$ by rational numbers and checks whether these rational approximations satisfy the constraints. In such a case they guarantee a certified duality gap. The interested reader can download rma from our web page [58].

\section{An LMI Constraint for the Inhomogeneous Minimum}

In this section we will give an LMI in the parameters $\left(q_{i j}\right)$ of a PQF $Q=\left(q_{i j}\right)$, which is satisfied if and only if the inhomogeneous minimum of $Q$ is bounded by a constant,

\footnotetext{
1 http: //www-user.tu-chemnitz. de/ helmberg/semidef.html.

2 http: //Www. stanford. edu/ boyd/MAXDET. html.
} 
say $\mu(Q) \leq 1$. For this it is crucial to observe that $\boldsymbol{x}^{t} Q \boldsymbol{y}$ is a linear expression in the parameters $\left(q_{i j}\right)$. The PQF $Q=\left(q_{i j}\right)$ defines the inner product of a Euclidean space $\left(\mathbb{R}^{d},(\cdot, \cdot)\right)$ by $(\boldsymbol{x}, \boldsymbol{y})=\boldsymbol{x}^{t} Q \boldsymbol{y}$.

From Section 3 of [27] we can extract the following proposition which is central in our further discussion.

Proposition 7.1. Let $L=\operatorname{conv}\left\{\boldsymbol{0}, \boldsymbol{v}_{1}, \ldots, \boldsymbol{v}_{d}\right\} \subseteq \mathbb{R}^{d}$ be ad-dimensional simplex. Then the radius of the circumsphere of $L$ is at most 1 with respect to $(\cdot, \cdot)$ if and only if the following linear matrix inequality (in the parameters $q_{i j}$ ) is satisfied:

$$
\mathrm{BR}_{L}(Q)=\left(\begin{array}{ccccc}
4 & \left(\boldsymbol{v}_{1}, \boldsymbol{v}_{1}\right) & \left(\boldsymbol{v}_{2}, \boldsymbol{v}_{2}\right) & \cdots & \left(\boldsymbol{v}_{d}, \boldsymbol{v}_{d}\right) \\
\left(\boldsymbol{v}_{1}, \boldsymbol{v}_{1}\right) & \left(\boldsymbol{v}_{1}, \boldsymbol{v}_{1}\right) & \left(\boldsymbol{v}_{1}, \boldsymbol{v}_{2}\right) & \cdots & \left(\boldsymbol{v}_{1}, \boldsymbol{v}_{d}\right) \\
\left(\boldsymbol{v}_{2}, \boldsymbol{v}_{2}\right) & \left(\boldsymbol{v}_{2}, \boldsymbol{v}_{1}\right) & \left(\boldsymbol{v}_{2}, \boldsymbol{v}_{2}\right) & \cdots & \left(\boldsymbol{v}_{2}, \boldsymbol{v}_{d}\right) \\
\vdots & \vdots & \vdots & \ddots & \vdots \\
\left(\boldsymbol{v}_{d}, \boldsymbol{v}_{d}\right) & \left(\boldsymbol{v}_{d}, \boldsymbol{v}_{1}\right) & \left(\boldsymbol{v}_{d}, \boldsymbol{v}_{2}\right) & \cdots & \left(\boldsymbol{v}_{d}, \boldsymbol{v}_{d}\right)
\end{array}\right) \succeq 0 .
$$

In [27] Delone et al. used this proposition as the key ingredient for showing that the set of PQFs which determine a circumsphere of $L$ with radius of at most $R$ is convex and bounded (see Proposition 9.1). Because of the importance of the proposition and for the convenience of the reader we give a short proof here.

Proof. We make use of Cayley-Menger determinants. The Cayley-Menger determinant of $d+1$ points $\boldsymbol{v}_{0}, \ldots, \boldsymbol{v}_{d}$ with pairwise distances $\operatorname{dist}\left(\boldsymbol{v}_{i}, \boldsymbol{v}_{j}\right)$ is

$$
\mathrm{CM}\left(\boldsymbol{v}_{0}, \ldots, \boldsymbol{v}_{d}\right)=\left|\begin{array}{cccc}
0 & 1 & \cdots & 1 \\
1 & \operatorname{dist}\left(\boldsymbol{v}_{0}, \boldsymbol{v}_{0}\right)^{2} & \cdots & \operatorname{dist}\left(\boldsymbol{v}_{0}, \boldsymbol{v}_{d}\right)^{2} \\
\vdots & \vdots & \ddots & \vdots \\
1 & \operatorname{dist}\left(\boldsymbol{v}_{d}, \boldsymbol{v}_{0}\right)^{2} & \cdots & \operatorname{dist}\left(\boldsymbol{v}_{d}, \boldsymbol{v}_{d}\right)^{2}
\end{array}\right|
$$

The squared circumradius $R^{2}$ of the simplex $L$ equals (see for example Proposition 9.7.3.7 of [17])

$$
R^{2}=-\frac{1}{2} \cdot \frac{\operatorname{det}\left(\operatorname{dist}\left(\boldsymbol{v}_{i}, \boldsymbol{v}_{j}\right)^{2}\right)_{0 \leq i, j \leq d}}{\operatorname{CM}\left(\boldsymbol{v}_{0}, \ldots, \boldsymbol{v}_{d}\right)}
$$

Replacing dist $(\boldsymbol{x}, \boldsymbol{y})^{2}$ by $(\boldsymbol{x}, \boldsymbol{x})-2(\boldsymbol{x}, \boldsymbol{y})+(\boldsymbol{y}, \boldsymbol{y})$, using $\boldsymbol{v}_{0}=\boldsymbol{0}$ and performing elementary transformations of the determinants turns the above formula into

$$
R^{2}=-\frac{1}{4} \cdot \frac{\left|\begin{array}{ccccc}
0 & \left(\boldsymbol{v}_{1}, \boldsymbol{v}_{1}\right) & \left(\boldsymbol{v}_{2}, \boldsymbol{v}_{2}\right) & \cdots & \left(\boldsymbol{v}_{d}, \boldsymbol{v}_{d}\right) \\
\left(\boldsymbol{v}_{1}, \boldsymbol{v}_{1}\right) & \left(\boldsymbol{v}_{1}, \boldsymbol{v}_{1}\right) & \left(\boldsymbol{v}_{1}, \boldsymbol{v}_{2}\right) & \cdots & \left(\boldsymbol{v}_{1}, \boldsymbol{v}_{d}\right) \\
\vdots & \vdots & \vdots & \ddots & \vdots \\
\left(\boldsymbol{v}_{d}, \boldsymbol{v}_{d}\right) & \left(\boldsymbol{v}_{d}, \boldsymbol{v}_{1}\right) & \left(\boldsymbol{v}_{d}, \boldsymbol{v}_{2}\right) & \cdots & \left(\boldsymbol{v}_{d}, \boldsymbol{v}_{d}\right)
\end{array}\right|}{\operatorname{det}\left(\left(\boldsymbol{v}_{i}, \boldsymbol{v}_{j}\right)\right)_{1 \leq i, j \leq d}}
$$


The inequality $R \leq 1$ is equivalent to

$$
4 \cdot \operatorname{det}\left(\left(\boldsymbol{v}_{i}, \boldsymbol{v}_{j}\right)\right)_{1 \leq i, j \leq d}+\left|\begin{array}{ccccc}
0 & \left(\boldsymbol{v}_{1}, \boldsymbol{v}_{1}\right) & \left(\boldsymbol{v}_{2}, \boldsymbol{v}_{2}\right) & \ldots & \left(\boldsymbol{v}_{d}, \boldsymbol{v}_{d}\right) \\
\left(\boldsymbol{v}_{1}, \boldsymbol{v}_{1}\right) & \left(\boldsymbol{v}_{1}, \boldsymbol{v}_{1}\right) & \left(\boldsymbol{v}_{1}, \boldsymbol{v}_{2}\right) & \ldots & \left(\boldsymbol{v}_{1}, \boldsymbol{v}_{d}\right) \\
\vdots & \vdots & \vdots & \ddots & \vdots \\
\left(\boldsymbol{v}_{d}, \boldsymbol{v}_{d}\right) & \left(\boldsymbol{v}_{d}, \boldsymbol{v}_{1}\right) & \left(\boldsymbol{v}_{d}, \boldsymbol{v}_{2}\right) & \ldots & \left(\boldsymbol{v}_{d}, \boldsymbol{v}_{d}\right)
\end{array}\right| \geq 0,
$$

which is equivalent to

$$
\left|\begin{array}{ccccc}
4 & \left(v_{1}, v_{1}\right) & \left(v_{2}, v_{2}\right) & \ldots & \left(v_{d}, v_{d}\right) \\
\left(v_{1}, v_{1}\right) & \left(v_{1}, v_{1}\right) & \left(v_{1}, v_{2}\right) & \ldots & \left(v_{1}, v_{d}\right) \\
\vdots & \vdots & \vdots & \ddots & \vdots \\
\left(v_{d}, v_{d}\right) & \left(v_{d}, v_{1}\right) & \left(v_{d}, v_{2}\right) & \ldots & \left(v_{d}, v_{d}\right)
\end{array}\right| \geq 0
$$

The minors in the lower right are all determinants of Gram matrices and therefore nonnegative. Hence, the matrix $\operatorname{BR}_{L}(Q)$ is positive semidefinite.

Corollary 7.2. For any d-dimensional simplex $L \subseteq \mathbb{R}^{d}$ with vertex at 0 and $Q \in \mathcal{S}_{>0}^{d}$ we have

$$
\left|\mathrm{BR}_{L}(Q)\right| \geq 0 \quad \Longleftrightarrow \quad \mathrm{BR}_{L}(Q) \succeq 0
$$

Example 7.3. Let us compute the matrix linear inequality $\mathrm{BR}_{L}(Q) \succeq 0, Q=\left(\begin{array}{ll}q_{11} & q_{21} \\ q_{21} & q_{22}\end{array}\right)$, for the two-dimensional simplex

$$
L=\operatorname{conv}\left\{\left(\begin{array}{l}
0 \\
0
\end{array}\right),\left(\begin{array}{l}
1 \\
0
\end{array}\right),\left(\begin{array}{l}
1 \\
1
\end{array}\right)\right\}
$$

We have

$$
\begin{aligned}
\mathrm{BR}_{L}(Q) & =\left(\begin{array}{ccc}
4 & q_{11} & q_{11}+2 q_{21}+q_{22} \\
q_{11} & q_{11} & q_{11}+q_{21} \\
q_{11}+2 q_{21}+q_{22} & q_{11}+q_{21} & q_{11}+2 q_{21}+q_{22}
\end{array}\right) \\
& =\left(\begin{array}{lll}
4 & 0 & 0 \\
0 & 0 & 0 \\
0 & 0 & 0
\end{array}\right)+q_{11}\left(\begin{array}{lll}
0 & 1 & 1 \\
1 & 1 & 1 \\
1 & 1 & 1
\end{array}\right)+q_{21}\left(\begin{array}{lll}
0 & 0 & 2 \\
0 & 0 & 1 \\
2 & 1 & 2
\end{array}\right)+q_{22}\left(\begin{array}{lll}
0 & 0 & 1 \\
0 & 0 & 0 \\
1 & 0 & 1
\end{array}\right)
\end{aligned}
$$

For a Delone polytope $L$ other than a simplex the circumradius is less than or equal to 1 if and only if it is less than or equal to 1 for some $d$-dimensional simplex $L^{\prime}$ with vertices in vert $L$. Therefore we set $\operatorname{BR}_{L}(Q)=\operatorname{BR}_{L^{\prime}}(Q)$ in this case. Since a block matrix is semidefinite if and only if the blocks are semidefinite, we have the following proposition which allows us to express the constraint " $\mu(Q) \leq 1$ " locally by a single LMI. 
Proposition 7.4. Let $Q=\left(q_{i j}\right) \in \mathcal{S}_{>0}^{d}$ be a PQF and let $L_{1}, \ldots, L_{n}$ be a representative system of all inequivalent d-dimensional Delone polytopes in $\operatorname{Del}(Q)$. Then

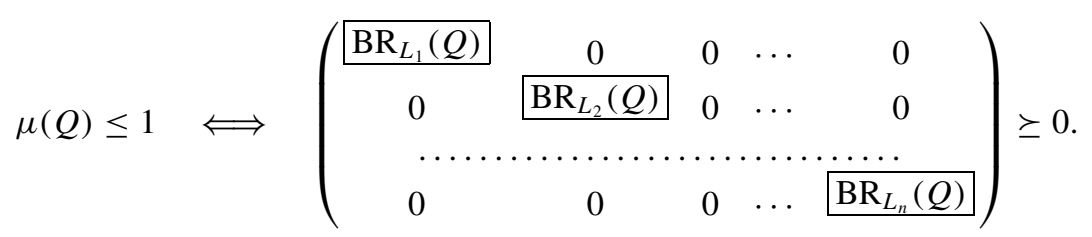

\section{Algorithms}

In this section we present algorithms which in theory solve the lattice covering problem and the lattice packing-covering problem in any dimension $d$.

Our algorithm for the lattice covering problem computes all locally optimal lattice coverings of a given dimension. These are only finitely many because we will see that for every fixed Delone triangulation $\mathcal{D}$ there exists at most one PQF lying in the topological closure of the secondary cone of $\mathcal{D}$ giving a locally optimal covering density. So, we fix a Delone triangulation and try to find the PQF which minimizes the density function in the topological closure of the secondary cone of the fixed Delone triangulation. We formulate this restricted lattice covering problem as a determinant maximization problem.

Our algorithm for the lattice packing-covering problem operates similarly. For every Delone triangulation we have to solve a semidefinite programming problem.

\subsection{Solving the Lattice Covering Problem}

Recall that the covering density of a PQF $Q$ in $d$ variables is given by $\Theta(Q)=$ $\sqrt{\mu(Q)^{d} / \operatorname{det} Q} \cdot \kappa_{d}$. Scaling of $Q$ by a positive real number $\alpha$ leaves $\Theta$ invariant. Consequently, we can restrict our attention to those PQFs $Q$ with $\mu(Q)=1$. Hence, we solve the lattice covering problem if we solve the optimization problem:

\begin{tabular}{|ll|}
\hline maximize & $\operatorname{det}(Q)$ \\
subject to & $Q$ is a positive definite matrix, \\
& $\mu(Q)=1$
\end{tabular}

where the optimization variables $q_{i j}$ are the entries of the PQF $Q$. The major disadvantage of this optimization problem is that the second constraint is not expressible as a convex condition in the variables $q_{i j}$ and that the problem has many local maxima. A locally optimal solution is also called a locally optimal lattice covering.

We circumvent this by splitting the original problem into a finite number of determinant maximization problems. For every Delone triangulation $\mathcal{D}$ we solve the optimization problem:

\begin{tabular}{|cc|}
\hline maximize & $\operatorname{det}(Q)$ \\
subject to & $Q \in \overline{\Delta(\mathcal{D})}$ \\
& $\mu(Q) \leq 1$ \\
\hline
\end{tabular}


The relaxation of no longer requiring $\mu(Q)=1$ in the third constraint does not give more optimal solutions because if $Q$ satisfies the constraints, then so does $(1 / \mu(Q)) Q$. Now, we have to show that this is indeed a determinant maximization problem. We have seen in Proposition 5.1 that the first constraint can be expressed with inequalities linear in $q_{i j}$. The constraint $\mu(Q) \leq 1$ can be expressed by an LMI as in Proposition 7.4.

The optimization vector $\boldsymbol{x} \in \mathbb{R}^{d(d+1) / 2}$ is the vector of coefficients of $Q$. The LMI $G(\boldsymbol{x}) \succ 0$ is given by $G(\boldsymbol{x})=Q$. We encode the two other constraints $Q \in \overline{\boldsymbol{\Delta}(Q)}$ and $\mu(Q) \leq 1$ by block matrices in the LMI $F(\boldsymbol{x}) \succeq 0$. For any linear inequality which is needed to describe the secondary cone we have a $1 \times 1$ block matrix. For any inequivalent $d$-dimensional simplex $L \in \mathcal{D}$ we have the $(d+1) \times(d+1)$ block matrix $\operatorname{BR}_{L}(Q)$.

\subsection{Solving the Lattice Packing-Covering Problem}

Along the same lines as above we formulate the lattice packing-covering problem as a finite number of semidefinite programming problems.

Recall that the packing-covering constant of a PQF $Q$ is $\gamma(Q)=2 \cdot \sqrt{\mu(Q) / \lambda(Q)}$. Since $\gamma$ is homogeneous we can again assume $\mu(Q)=1$, and the lattice packingcovering problem is equivalent to the following optimization problem:

$\begin{array}{ll}\text { maximize } & \lambda(Q) \\ \text { subject to } & Q \text { is a positive definite matrix, } \\ & \mu(Q)=1\end{array}$

A locally optimal solution of the optimization problem is called a locally optimal lattice packing-covering.

From the previous discussion we know how to deal with the constraint $\mu(Q)=1$. Now, how do we maximize $\lambda(Q)$ ? We say that $\boldsymbol{v} \in \mathbb{Z}^{d} \backslash\{\boldsymbol{0}\}$ is a shortest vector of $Q$ if $Q[v]=\lambda(Q)$. A theorem of Voronoi (see Section 55 of [64] or Theorem 10 in Chapter 21 of [22]) implies that a shortest vector $\boldsymbol{v}$ gives the edge $[\boldsymbol{0}, \boldsymbol{v}]$ in the Delone subdivision of $Q$. In a fixed Delone subdivision $\mathcal{D}$ there are only finitely many (at most $2\left(2^{d}-1\right)$, see Section 55 of [64]) edges of the form [0,v]. We can maximize $\lambda(Q)$ for $Q \in \overline{\Delta(\mathcal{D})}$ as follows: We introduce a new variable $C$ which we want to maximize subject to the constraints $Q[\boldsymbol{v}] \geq C$ where $\boldsymbol{v}$ runs through all edges of the form $[\boldsymbol{0}, \boldsymbol{v}]$ in $\mathcal{D}$. This assures $\lambda(Q)=C$ when $C$ attains a maximum. All these expressions are linear in the coefficients $q_{i j}$ of $Q$. Hence we have to solve the following semidefinite programming problem for every inequivalent Delone triangulation $\mathcal{D}$ in order to solve the lattice packing-covering problem:

\begin{tabular}{|ll|}
\hline maximize & $C$ \\
subject to & $Q \in \overline{\boldsymbol{\Delta}(\mathcal{D})}$ \\
& $\mu(Q) \leq 1$, \\
& $Q[\boldsymbol{v}] \geq C$, where $[\boldsymbol{0}, \boldsymbol{v}]$ is an edge in $\mathcal{D}$. \\
\hline
\end{tabular}




\section{Local Optima}

Since we are dealing with convex optimization problems, we can extract some structural information about uniqueness and invariance properties of locally optimal solutions. On the one hand these help us to identify exact coordinates of optimal solutions. On the other hand they allow us to decide whether we have found an isolated locally optimal solution or not.

\subsection{Properties of Local Optima}

Most of the desired properties follow from the special structure of sets with PQFs $Q$ attaining certain fixed values $\mu(Q), \lambda(Q)$ or $\operatorname{det}(Q)$. Let $L=\operatorname{conv}\left\{\boldsymbol{0}, \boldsymbol{v}_{1}, \ldots, \boldsymbol{v}_{d}\right\} \subseteq \mathbb{R}^{d}$ denote a $d$-dimensional simplex and let $\mathcal{D}$ denote a Delone triangulation. Then we consider the sets

$$
\begin{aligned}
\mathbf{V}_{L} & =\left\{Q \in \mathcal{S}_{>0}^{d}:\left|\operatorname{BR}_{L}(Q)\right| \geq 0\right\}, \\
\widehat{\mathbf{V}}_{L} & =\left\{Q \in \mathcal{S}_{>0}^{d}:\left|\operatorname{BR}_{L}(Q)\right|=0\right\}, \\
\mathbf{W}_{\mathcal{D}} & =\bigcap_{L \in \mathcal{D}} \mathbf{V}_{L} \cap \boldsymbol{\Delta}(\mathcal{D}) .
\end{aligned}
$$

Hence, by Corollary 7.2 and Proposition 7.4 the PQFs $Q \in \mathbf{W}_{\mathcal{D}}$ are those with $\mu(Q) \leq 1$ in $\Delta(\mathcal{D})$. Further, for $D>0$ and $\lambda>0$ we consider

$$
\begin{aligned}
& \mathbf{D}_{D}=\left\{Q \in \mathcal{S}_{>0}^{d}: \operatorname{det}(Q) \geq D\right\}, \\
& \mathbf{M}_{\lambda}=\left\{Q \in \mathcal{S}_{>0}^{d}: \lambda(Q) \geq \lambda\right\} .
\end{aligned}
$$

The following is known (see [27] and [52]) and was partially discussed in previous sections:

Proposition 9.1. Let $L=\operatorname{conv}\left\{\boldsymbol{0}, \boldsymbol{v}_{1}, \ldots, \boldsymbol{v}_{d}\right\} \subseteq \mathbb{R}^{d}$ be a d-dimensional simplex, let $\mathcal{D}$ be a Delone triangulation, let $D>0$ and let $\lambda>0$. Then

1. $\mathbf{V}_{L}$, and therefore $\mathbf{W}_{\mathcal{D}}$, is convex and bounded.

2. $\widehat{\mathbf{V}}_{L}$ is a smooth (regular) hypersurface.

3. $\mathbf{D}_{D}$ is unbounded, strictly convex and has a smooth boundary.

4. $\mathbf{M}_{\lambda}$ is unbounded and convex and has a piecewise linear boundary.

5. $\mathbf{D}_{D}$ and $\mathbf{M}_{\lambda}$ are invariant with respect to the action of $\mathrm{GL}_{d}(\mathbb{Z})$ on $\mathcal{S}_{>0}^{d}$.

As a consequence of these properties, it is not hard to derive the following proposition (see [27] and [52]).

Proposition 9.2. Let $\mathcal{D}$ be a Delone triangulation.

1. The set of $P Q F$ s in $\overline{\boldsymbol{\Delta}(\mathcal{D})}$ attaining $\min _{Q \in \overline{\boldsymbol{\Delta}(\mathcal{D})}} \Theta(Q)$ is a single $P Q F$, together with all of its positive multiples. It is invariant with respect to the action of $\mathcal{D}$ 's 
automorphism group

$$
\operatorname{Aut}(\mathcal{D})=\left\{U \in \mathrm{GL}_{d}(\mathbb{Z}): \mathcal{D} U=\mathcal{D}\right\} .
$$

2. The set of $P Q F$ s in $\overline{\Delta(\mathcal{D})}$ attaining $\min _{Q \in \overline{\Delta(\mathcal{D})}} \gamma(Q)$ is convex and contains a subset which is invariant with respect to $\operatorname{Aut}(\mathcal{D})$.

A first, but non-geometric proof of property 1 was given by Barnes and Dickson [15]. There they also observed, as Ryshkov [52] did for the lattice packing-covering problem, that it suffices to optimize among all PQFs whose automorphism group contains the $\operatorname{group} \operatorname{Aut}(\mathcal{D})$.

Corollary 9.3. If $Q$ is a local optimum of the lattice covering or lattice packingcovering problem among all PQFs in $\Delta(\mathcal{D})$ whose automorphism group contains $\operatorname{Aut}(\mathcal{D})$, then $Q$ is a locally optimal solution.

Note that the statement above holds only if $Q$ lies in the interior of the secondary cone. For local optima on the boundary of secondary cones we may apply the following trivial proposition.

Proposition 9.4. A $P Q F Q$ is a locally optimal solution with respect to $\Theta$ or $\gamma$ if and only if it is an optimal solution for all Delone triangulations $\mathcal{D}$ with $Q \in \overline{\Delta(\mathcal{D})}$.

There probably exist two locally optimal solutions with respect to $\gamma$ in dimension 5 which both lie on the boundary of some secondary cones (see Section 11, proof of Theorem 11.2). In dimension 5 and lower every locally optimal solution with respect to $\Theta$ lies in the interior of some secondary cone. However, in higher dimensions there exist locally optimal solutions lying on the boundary. The Leech lattice gives such an example as recently shown by the authors in [57].

\subsection{Rigorous Certificates}

Using convex optimization software to solve the covering or packing-covering problem, we are often limited to determining a certain certified range (see Section 6) in which the optimum value is attained. To use Proposition 9.4 it is desirable to decide computationally whether or not the optimum is attained on the boundary or even on a specific facet of $\overline{\Delta(\mathcal{D})}$. In many cases this is possible by using the following proposition which is a simple consequence of the convexity of determinant maximization problems.

Proposition 9.5. Consider the determinant maximization problem

$$
\begin{array}{ll}
\text { minimize } & f(\boldsymbol{x}) \\
\text { subject to } & G(\boldsymbol{x}) \succ 0 \text { and } F(\boldsymbol{x}) \succeq 0,
\end{array}
$$

where $f(\boldsymbol{x})=\boldsymbol{c}^{t} \boldsymbol{x}-\log \operatorname{det} G(\boldsymbol{x})$.

1. Let $\boldsymbol{y}_{1}$ be the optimal solution of (4), and let $\boldsymbol{y}_{2}$ be the optimal solution of (4) with the additional constraint $\tilde{F}(\boldsymbol{x}) \succeq 0$. If $f\left(\boldsymbol{y}_{1}\right)<f\left(\boldsymbol{y}_{2}\right)$, then $\tilde{F}\left(\boldsymbol{y}_{2}\right)=0$. 
2. Let $\boldsymbol{y}_{1}$ be an optimal solution of (4) with the additional linear constraint $\boldsymbol{n}^{t} \boldsymbol{x} \geq 0$, and let $\boldsymbol{y}_{2}$ be an optimal solution of (4) with the additional linear constraint $\boldsymbol{n}^{t} \boldsymbol{x} \leq 0$.

(a) If $f\left(\boldsymbol{y}_{1}\right)<f\left(\boldsymbol{y}_{2}\right)$, then $\boldsymbol{n}^{t} \boldsymbol{y}_{2}=0$.

(b) If $f\left(\boldsymbol{y}_{1}\right)>f\left(\boldsymbol{y}_{2}\right)$, then $\boldsymbol{n}^{t} \boldsymbol{y}_{2}<0$.

Let $Q_{\text {opt }}$ denote a PQF with $\mu\left(Q_{\text {opt }}\right)=1$ attaining an optimum in $\overline{\boldsymbol{\Delta ( \mathcal { D } )}}$ for $\Theta$. The lattice packing-covering case is similar. Let $l$ and $u$ be a lower and an upper bound for a minimum of $f(Q)=-\log \operatorname{det}(Q)$. Here and in what follows we use $\mu_{\mathcal{D}}(Q) \leq 1$ as an abbreviation for the LMI in Proposition 7.4 guaranteeing that the circumradius (with respect to $Q$ ) of all the simplices of the triangulation $\mathcal{D}$ is at most 1 .

The first part of Proposition 9.5 gives a sufficient criterion for $Q_{\text {opt }}$ lying on the boundary of $\overline{\boldsymbol{\Delta}(\mathcal{D})}$. Suppose the upper bound of $f$ on $\left\{Q \in \mathcal{S}_{>0}^{d}: \mu_{\mathcal{D}}(Q) \leq 1\right\}$ is smaller than $l$. Then we have a certificate for $Q_{\text {opt }} \in$ bd $\overline{\Delta(\mathcal{D})}$.

The first half of the second part of Proposition 9.5 gives a sufficient criterion for $Q_{\mathrm{opt}}$ lying on a specific facet $F$ of $\overline{\Delta(\mathcal{D})}$. Let $H$ denote a hyperplane containing $F$ and let $H^{-}, H^{+}$be the closed halfspaces containing $\overline{\Delta(\mathcal{D})}$, respectively not containing $\overline{\Delta(\mathcal{D})}$. If the upper bound of $f$ on $\left\{Q \in \mathcal{S}_{>0}^{d}: Q \in H^{+}\right.$and $\left.\mu_{\mathcal{D}}(Q) \leq 1\right\}$ is smaller than $l$, then we have a certificate for $Q_{\text {opt }} \in F$.

The second half of the second part of Proposition 9.5 gives a sufficient criterion for $Q_{\text {opt }}$ not lying on a specific facet $F$ of $\overline{\boldsymbol{\Delta}(\mathcal{D})}$. If the lower bound of $f$ on $\{Q \in$ $\mathcal{S}_{>0}^{d}: Q \in H^{+}$and $\left.\mu_{\mathcal{D}}(Q) \leq 1\right\}$ is larger than $u$, then we have a certificate for $Q_{\text {opt }} \notin F$. Clearly, such a certificate for all facets of $\overline{\Delta(\mathcal{D})}$ gives a certificate for $Q_{\text {opt }} \in \Delta(\mathcal{D})$.

Note that these certificates give mathematical rigorous proofs when we proceed as described at the end of Section 6.

\subsection{Necessary and Sufficient Conditions}

Next we assume a PQF $Q$ is given and we want to decide computationally if it is a locally optimal solution to the lattice covering or lattice packing-covering problem. In Section 9.3.2 we consider the case when $Q$ lies in the interior of the secondary cone of some Delone triangulation. In this case we give necessary and sufficient conditions for $Q$ being a locally optimal solution. The case when $Q$ lies on the boundary of the secondary cone of some Delone triangulation is more subtle and we deal with it in Section 9.3.3.

9.3.1. Normal Cones. Before considering locally optimal solutions we have to do some local analysis. Here, we shall compute the normal cones of the sets $\mathbf{W}_{\mathcal{D}}, \mathbf{M}_{\lambda}$ and $\mathbf{D}_{D}$ at a given $\mathrm{PQF} Q$. As a general reference to the basic concepts in convex and differential geometry used in what follows we refer to the book [56] by Schneider.

We consider $\mathcal{S}^{d}$ as a Euclidean space with inner product $\langle\cdot, \cdot \cdot\rangle$. Let $H=\{S \in$ $\left.\mathcal{S}^{d}:\langle N, S\rangle=\alpha\right\}$ be a hyperplane with normal vector $N \in \mathcal{S}^{d} \backslash\{\boldsymbol{O}\}$ and let $C$ be a convex set with boundary point $Q$. Then $H$ is said to be a supporting hyperplane of $C$ at $Q$ with outer normal vector $N$, if $C \subseteq H^{-}=\left\{S \in \mathcal{S}^{d}:\langle N, S\rangle \leq\langle N, Q\rangle\right\}$. The normal cone of $C$ at $Q$ is then given by all outer normal vectors of supporting hyperplanes at $Q$ 
together with the zero vector. Clearly, the normal cones are convex. A hyperplane $H$ is called a separating hyperplane for two convex sets with a common boundary point $Q$, if it is a supporting hyperplane of both sets, but with opposite outer normal vectors. Such a hyperplane exists if and only if the corresponding normal cones $\mathcal{N}_{1}$ and $\mathcal{N}_{2}$ at $Q$ satisfy $-\mathcal{N}_{1} \cap \mathcal{N}_{2} \neq \varnothing$.

We now compute the normal cones of the sets $\mathbf{W}_{\mathcal{D}}, \mathbf{M}_{\lambda}$ and $\mathbf{D}_{D}$ at some PQF $Q$.

Proposition 9.6. Let $\mathcal{D}$ be a Delone triangulation and let $Q \in \mathbf{W}_{\mathcal{D}}$ be a PQF with $\mu(Q)=1$. Then the normal cone of $\mathbf{W}_{\mathcal{D}}$ at $Q$ is equal to $-\operatorname{cone}\left\{g_{L}(Q): L \in \mathcal{D}\right.$ and $Q \in$ $\left.\widehat{\mathbf{V}}_{L}\right\}$, where $g_{L}(Q)=\operatorname{grad}\left|\mathrm{BR}_{L}\right|(Q)$ denotes the gradient of the regular surface $\widehat{\mathbf{V}}_{L}$ at $Q$.

Proof. First, $-g_{L}(Q) \neq \mathbf{0}$ exists and is the unique outer normal vector of $\mathbf{V}_{L}$ at $Q$, since $\widehat{\mathbf{V}}_{L}$ is a regular surface defined by the polynomial equation $\left|\mathrm{BR}_{L}(Q)\right|=0$. Second, we see that $\mathbf{W}_{\mathcal{D}}$, in a sufficiently small neighborhood of $Q$, is equal to the intersection $\bigcap_{Q \in \widehat{\mathbf{V}}_{L}} \mathbf{V}_{L}$. Therefore, the normal cone of $\mathbf{W}_{\mathcal{D}}$ at $Q$ is equal to cone $\left\{-g_{L}(Q): Q \in \widehat{\mathbf{V}}_{L}\right\}$ (see Theorem 2.2.1 of [56]) and the assertion follows.

By Proposition 9.1 the boundary of $\mathbf{D}_{\operatorname{det}(Q)}$ is smooth at $Q$, hence up to scaling there is a uniquely determined outer normal. The following is well known (see Proposition 10.3 and its detailed proof in [62, Proposition 8.2.2]).

Proposition 9.7. The normal cone of $\mathbf{D}_{\operatorname{det}(Q)}$ at $Q$ is given by $-\operatorname{cone}\left\{Q^{-1}\right\}$.

The normal cone of $\mathbf{M}_{\lambda}$ at a boundary point $Q$ is determined by the shortest vectors of $Q$, that is, by those $\boldsymbol{v} \in \mathbb{Z}^{d}$ for which the homogeneous minimum $\lambda(Q)$ is attained. Recall that $Q[\boldsymbol{v}]=\lambda$ for a fixed $\boldsymbol{v} \in \mathbb{Z}^{d} \backslash\{\boldsymbol{0}\}$ is linear in the parameters $q_{i j}$, hence this condition defines a hyperplane in $\mathcal{S}^{d}$.

Proposition 9.8. For $\boldsymbol{v} \in \mathbb{Z}^{d} \backslash\{\boldsymbol{0}\}$ let $V_{\boldsymbol{v}} \in \mathcal{S}^{d}$ be the normal vector of the hyperplane $\left\{Q^{\prime} \in \mathcal{S}^{d}: Q^{\prime}[\boldsymbol{v}]=\lambda(Q)\right\}$ with $\left\langle Q, V_{v}\right\rangle=\lambda(Q)$. Then the normal cone of $\mathbf{M}_{\lambda}$ at $Q$ is given by $-\operatorname{cone}\left\{V_{v}: Q[v]=\lambda(Q)\right\}$.

Proof. This follows immediately from Theorem 2.2.1 of [56].

Note that a normal vector $V_{v}$ depends on the chosen inner product. For example for practical computations it is convenient to identify $\mathcal{S}^{d}$ with $\mathbb{R}^{d(d+1) / 2}$ and to use the standard inner product $\langle Q, V\rangle=\sum_{i \leq j} q_{i j} v_{i j}$ and $V_{\boldsymbol{v}}=\left(v_{i j}\right)$ with $v_{i j}=\left(2-\delta_{i j}\right) \boldsymbol{v}_{i} \boldsymbol{v}_{j}$.

9.3.2. Interior Cases. Because of the convexity of $\mathbf{D}_{\operatorname{det}(Q)}, \mathbf{M}_{\lambda(Q)}$ and $\mathbf{W}_{\mathcal{D}}$ a separating hyperplane at a PQF $Q$ of $\mathbf{W}_{\mathcal{D}}$ with $\mu(Q)=1$ and one of the other two sets yields a necessary and sufficient condition for $Q$ to be a locally optimal solution to either the lattice covering or the lattice packing-covering problem. Therefore a necessary and sufficient condition can be derived from the normal cones at $Q$ with respect to these sets. 
Proposition 9.9. Let $\mathcal{D}$ be a Delone triangulation, and let $Q \in \Delta(\mathcal{D})$ be a PQF with $\mu(Q)=1$.

1. [15] Then $Q$ is a locally optimal solution to the lattice covering problem if and only if

$$
\text { cone }\left\{Q^{-1}\right\} \cap-\operatorname{cone}\left\{g_{L}(Q): L \in \mathcal{D} \text { and } Q \in \widehat{\mathbf{V}}_{L}\right\} \neq \emptyset .
$$

2. Then $Q$ is a locally optimal solution to the lattice packing-covering problem if and only if

$$
\text { cone }\left\{V_{v}:\left\langle Q, V_{v}\right\rangle=\lambda(Q)\right\} \cap-\operatorname{cone}\left\{g_{L}(Q): L \in \mathcal{D} \text { and } Q \in \widehat{\mathbf{V}}_{L}\right\} \neq \emptyset .
$$

9.3.3. Boundary Cases. Note that the foregoing proposition does not give criteria for PQFs $Q$ on the boundary of some secondary cones. In such a case we need to replace - cone $\left\{g_{L}(Q): L \in \mathcal{D}\right.$ and $\left.Q \in \widehat{\mathbf{V}}_{L}\right\}$ by a generalized expression. That is done by considering for each Delone triangulation $\mathcal{D}$ with $Q \in \overline{\Delta(\mathcal{D})}$ the normal cone $\mathcal{C}_{\mathcal{D}}(Q)$ of $\overline{\mathbf{W}_{\mathcal{D}}}$ at $Q$. We have $\mathcal{C}_{\mathcal{D}}(Q)=-\operatorname{cone}\left(\mathcal{N}_{1} \cup \mathcal{N}_{2}\right)$ with outer normal vectors $\mathcal{N}_{1}=$ cone $\left\{g_{L}(Q): L \in \mathcal{D}\right.$ and $\left.Q \in \widehat{\mathbf{V}}_{L}\right\}$, as considered before, and outer normal vectors

$$
\begin{aligned}
\mathcal{N}_{2}= & \left\{N \in \mathcal{S}^{d}:\langle N, \cdot\rangle=\varrho_{\left(L, L^{\prime}\right)}(\cdot) \text { for a pair }\left(L, L^{\prime}\right)\right. \text { of adjacent simplices } \\
& \text { of } \left.\mathcal{D} \text { with } \varrho_{\left(L, L^{\prime}\right)}(Q)=0\right\}
\end{aligned}
$$

from facets of $\overline{\boldsymbol{\Delta}(\mathcal{D})}$ containing $Q$. Since $\mathbf{D}_{D}$ has a smooth boundary, a necessary condition for a local covering optimum at $Q$ is the convexity of $\bigcup_{Q \in \overline{\Delta(\mathcal{D})}} \overline{\mathbf{W}_{\mathcal{D}}}$ at $Q$ which is equivalent to $\bigcap_{Q \in \overline{\Delta(\mathcal{D})}} \mathcal{C}_{\mathcal{D}}(Q) \neq \emptyset$. In case of the lattice packing-covering problem, this convexity condition is not necessary for a local optimum. The following proposition however gives sufficient conditions for a local optimum in case the convexity condition is satisfied.

Proposition 9.10. Let $Q \in \mathcal{S}_{>0}^{d}$ be a $P Q F$ with $\mu(Q)=1$.

1. Then $Q$ is a locally optimal solution to the lattice covering problem if and only if

$$
\operatorname{cone}\left\{Q^{-1}\right\} \cap \bigcap_{Q \in \overline{\Delta(\mathcal{D})}} \mathcal{C}_{\mathcal{D}}(Q) \neq \emptyset .
$$

2. Then $Q$ is a locally optimal solution to the lattice packing-covering problem if

$$
\text { cone }\left\{V_{v}:\left\langle Q, V_{v}\right\rangle=\lambda(Q)\right\} \cap \bigcap_{Q \in \overline{\Delta(\mathcal{D})}} \mathcal{C}_{\mathcal{D}}(Q) \neq \emptyset .
$$

Proof. The set $\bigcap_{Q \in \overline{\Delta(\mathcal{D})}} \mathcal{C}_{\mathcal{D}}(Q)$ contains all common outer normal vectors of the sets $\overline{\mathbf{W}_{\mathcal{D}}}$ with $Q \in \overline{\Delta(\mathcal{D})}$ at $Q$. In particular, $\bigcap_{Q \in \overline{\Delta(\mathcal{D})}} \mathcal{C}_{\mathcal{D}}(Q)$ is empty if and only if $\bigcup_{Q \in \overline{\boldsymbol{\Delta}(\mathcal{D})}} \overline{\mathbf{W}_{\mathcal{D}}}$ is not convex at $Q$. If convex, then a separating hyperplane of $\bigcup_{Q \in \overline{\boldsymbol{\Delta}(\mathcal{D})}} \overline{\mathbf{W}}_{\mathcal{D}}$ and $\mathbf{D}_{\operatorname{det}(Q)}$, respectively $\mathbf{M}_{\lambda(Q)}$, at $Q$ is sufficient for a local optimum at $Q$. The assertion then follows by Propositions 9.7 and 9.8 . 


\subsection{Conditions for Isolated Local Optima}

Locally optimal solutions to the lattice packing-covering problem may not be isolated optima, in contrast to solutions to the lattice covering problem. To determine computationally if a given locally optimal solution $Q$ is isolated, we have to check if there exists a segment $\left[Q, Q^{\prime}\right]$ which lies in the boundary of $\mathbf{M}_{\lambda(Q)}$ and in the boundary of $\mathbf{W}_{\mathcal{D}}$ at $Q$.

Proposition 9.11. Let $\mathcal{D}$ be a Delone triangulation and let $Q \in \Delta(\mathcal{D})$ be a locally optimal solution to the lattice packing-covering problem with $\mu(Q)=1$. Then $Q$ is not an isolated local optimum if and only if there exists an $S \in \mathcal{S}^{d}$ with

1. $\left\langle V_{\boldsymbol{v}}, S\right\rangle \geq 0$ for all $\boldsymbol{v} \in \mathbb{Z}^{d}$ with $Q[\boldsymbol{v}]=\lambda(Q)$,

2. $\left\langle g_{L}(Q), S\right\rangle \geq 0$ for all $L \in \mathcal{D}$ with $Q \in \widehat{\mathbf{V}}_{L}$,

3. For the Hessian $h_{L}(Q)=$ hess $\left|\mathrm{BR}_{L}\right|(Q)$ we have $h_{L}(Q) S=0$ for all $L \in \mathcal{D}$ with $Q \in \widehat{\mathbf{V}}_{L}$ and $\left\langle g_{L}(Q), S\right\rangle=0$.

Proof. The first condition says that there exists an $\varepsilon_{1}>0$ so that the segment $[Q, Q+$ $\left.\varepsilon_{1} S\right]$ lies in $\mathbf{M}_{\lambda(Q)}$ because $\mathbf{M}_{\lambda(Q)}$ has a piecewise linear boundary. The first condition together with the second condition plus the local optimality of $Q$ are equivalent to the fact that $S$ is in the tangent space of $\mathbf{V}_{L}$ at $Q$ and in the tangent space of $\mathbf{M}_{\lambda(Q)}$ at $Q$. Thus $Q+\varepsilon_{2} S$ is in the boundary of $\mathbf{W}_{\mathcal{D}}$ for sufficiently small $\varepsilon_{2}>0$ if and only if the corresponding smooth hypersurfaces $\widehat{\mathbf{V}}_{L}$ have curvature 0 in direction $S$. This is equivalent to $S$ being an eigenvector of eigenvalue 0 of the Hessian $h_{L}(Q)=$ hess $\left|\mathrm{BR}_{L}\right|(Q)$ which is the third condition. Hence, all three conditions are fulfilled if and only if there exists a segment $\left[Q, Q^{\prime}\right]$ in the boundary of the two sets $\mathbf{M}_{\lambda(Q)}$ and $\mathbf{W}_{\mathcal{D}}$, where $Q^{\prime}=Q+\min \left(\varepsilon_{1}, \varepsilon_{2}\right) S$.

Note again that the condition applies only if $Q$ is in the interior of a secondary cone. A similar sufficient condition for PQFs on the boundary of secondary cones can be attained by replacing $g_{L}(Q)$ in conditions 2 and 3 with the rays of the normal cone $\bigcap_{Q \in \overline{\boldsymbol{\Delta}(\mathcal{D})}} \mathcal{C}_{\mathcal{D}}(Q)$ of $\bigcup_{Q \in \overline{\boldsymbol{\Delta}(\mathcal{D})}} \overline{\mathbf{W}_{\mathcal{D}}}$ at $Q$ (see Proposition 9.10), if such exist.

Finally, we propose two (as far as we know) still unanswered questions.

Question 9.12. Does there exist a locally optimal solution to the lattice packingcovering problem which is not isolated?

Note that a positive answer to the following question would imply the existence of non-isolated locally optimal solutions.

Question 9.13. Does there exist a (locally optimal) solution to the lattice-packing covering problem, which does not have all the symmetries of the corresponding Delone subdivision? 


\section{Local Lower Bounds via Moments of Inertia}

In this section we give simple and efficiently computable local lower bounds for the lattice covering density and the lattice packing-covering constant. These bounds are "local" in the sense that they only apply to those PQFs lying in the topological closure of the secondary cone of a given Delone triangulation. For their computation we only need to know the coordinates of the simplices of the considered Delone triangulation. They are therefore useful tools in a heuristic search for "good" PQFs. The method goes back to Ryshkov and Delone. It is called the method of the moments of inertia because the central idea in its proof is analogous to the Parallel Axis Theorem of Steiner in classical mechanics. This analogy is explained in [3].

Let $P \subseteq \mathbb{R}^{d}$ be a finite set of points in $d$-dimensional Euclidean space $\left(\mathbb{R}^{d},(\cdot, \cdot)\right)$. We interpret the points of $P$ as masses with unit weight. The moment of inertia of the points about a point $\boldsymbol{x} \in \mathbb{R}^{d}$ is defined as $I_{x}(P)=\sum_{\boldsymbol{v} \in P} \operatorname{dist}(\boldsymbol{x}, \boldsymbol{v})^{2}$. The centroid of $P$ (center of gravity) is given by $\boldsymbol{m}=(1 /|P|) \sum_{\boldsymbol{v} \in P} \boldsymbol{v}$. From the equations

$$
\begin{aligned}
\operatorname{dist}(\boldsymbol{x}, \boldsymbol{v})^{2} & =(\boldsymbol{x}-\boldsymbol{v}, \boldsymbol{x}-\boldsymbol{v}) \\
& =(\boldsymbol{x}-\boldsymbol{m}, \boldsymbol{x}-\boldsymbol{m})+(\boldsymbol{m}-\boldsymbol{v}, \boldsymbol{m}-\boldsymbol{v})+2(\boldsymbol{x}-\boldsymbol{m}, \boldsymbol{m}-\boldsymbol{v}) \\
& =\operatorname{dist}(\boldsymbol{x}, \boldsymbol{m})^{2}+\operatorname{dist}(\boldsymbol{m}, \boldsymbol{v})^{2}+2(\boldsymbol{x}-\boldsymbol{m}, \boldsymbol{m}-\boldsymbol{v})
\end{aligned}
$$

and $\sum_{\boldsymbol{v} \in P}(\boldsymbol{m}-\boldsymbol{v})=\boldsymbol{0}$, we derive Apollonius' formula (see Section 9.7.6 of [17])

$$
I_{x}(P)=|P| \operatorname{dist}(\boldsymbol{x}, \boldsymbol{m})^{2}+I_{\boldsymbol{m}}(P) .
$$

Hence, the moment of inertia of $P$ about the centroid $\boldsymbol{m}$ is minimal.

If the points of $P$ form the vertices of a $d$-dimensional simplex, then (5) gives a relationship between the radius of the circumsphere $R$, the center of the circumsphere $\boldsymbol{c}$, and the moment of inertia about the centroid $\boldsymbol{m}$ of $P$ :

$$
R^{2}=\frac{I_{c}(P)}{d+1}=\operatorname{dist}(\boldsymbol{c}, \boldsymbol{m})^{2}+\frac{I_{\boldsymbol{m}}(P)}{d+1} .
$$

We can compute $I_{m}(P)$ using only the edge lengths of the simplex $P$. For every $\boldsymbol{w} \in \operatorname{vert} P$ we have by definition $I_{w}(P)=\sum_{\boldsymbol{v} \in P} \operatorname{dist}(\boldsymbol{w}, \boldsymbol{v})^{2}$. Summing up and using (5) gives

$$
\sum_{w \in P} I_{w}(P)=\sum_{w \in P}\left((d+1) \operatorname{dist}(\boldsymbol{w}, \boldsymbol{m})^{2}+I_{\boldsymbol{m}}(P)\right)=2(d+1) I_{\boldsymbol{m}}(P) .
$$

So, we get

$$
I_{\boldsymbol{m}}(P)=\frac{1}{d+1} \sum_{\{\boldsymbol{v}, \boldsymbol{w}\} \subseteq P} \operatorname{dist}(\boldsymbol{v}, \boldsymbol{w})^{2} .
$$

Let $\mathcal{D}$ be a Delone triangulation of $\mathbb{R}^{d}$, let $L_{1}, \ldots, L_{n}$ be the $d$-dimensional simplices of the star of a lattice point (say for example the origin), and let $\boldsymbol{m}_{i}$ be the centroid of $L_{i}, i=1, \ldots, n$. The arithmetical mean of the moments of inertia about the centroids of $L_{i}$ with respect to a PQF $Q$ is defined to be

$$
I_{\mathcal{D}}(Q)=\frac{1}{n} \sum_{i=1}^{n} I_{\boldsymbol{m}_{i}}\left(L_{i}\right)
$$


and is called the central moment of inertia of $\mathcal{D}$ with respect to $Q$. Note that we are now dealing with the inner product given by $Q$ with $\operatorname{dist}(\boldsymbol{x}, \boldsymbol{y})^{2}=Q[\boldsymbol{x}-\boldsymbol{y}]$ and that $I_{\mathcal{D}}(Q)$ is linear in the parameters $q_{i j}$ of $Q$.

Proposition 10.1. The central moment of inertia of $\mathcal{D}$ with respect to $Q$ yields a lower bound for the inhomogeneous minimum of $Q$ if $\mathcal{D}$ is a refinement of $\operatorname{Del}(Q)$. In this case we have

$$
\mu(Q) \geq \frac{1}{d+1} I_{\mathcal{D}}(Q)
$$

Proof. Let $R_{i}$ be the radius and let $\boldsymbol{c}_{i}$ be the center of the circumsphere of the simplex $L_{i}$, then

$$
\begin{aligned}
\mu(Q) & =\max _{i=1, \ldots, n} R_{i}^{2}=\max _{i=1, \ldots, n}\left(\operatorname{dist}\left(\boldsymbol{c}_{i}, \boldsymbol{m}_{i}\right)^{2}+\frac{I_{\boldsymbol{m}_{i}}\left(L_{i}\right)}{d+1}\right) \\
& \geq \max _{i=1, \ldots, n} \frac{I_{\boldsymbol{m}_{i}}\left(L_{i}\right)}{d+1} \geq \frac{1}{(d+1) n} \sum_{i=1}^{n} I_{\boldsymbol{m}_{i}}\left(L_{i}\right) \\
& =\frac{1}{d+1} I_{\mathcal{D}}(Q) .
\end{aligned}
$$

To find lower bounds for the lattice covering density or for the lattice packing-covering constant, we have to minimize the linear function $I_{\mathcal{D}}$ over all PQFs with a fixed determinant, or with a fixed homogeneous minimum, respectively. As in previous sections, we consider $\mathcal{S}^{d}$ as a Euclidean space with inner product $\langle\cdot, \cdot\rangle$.

Proposition 10.2. There exists a $P Q F F \in \mathcal{S}_{>0}^{d}$ with $I_{\mathcal{D}}=\langle F, \cdot\rangle$.

Proof. Since $I_{\mathcal{D}}$ is a linear function, there is an $F \in \mathcal{S}^{d}$ with $I_{\mathcal{D}}(\cdot)=\langle F, \cdot\rangle$. For every PQF $Q$, we have $\langle F, Q\rangle=I_{\mathcal{D}}(Q)>0$. Since $\mathcal{S}_{>0}^{d}=\left\{Q^{\prime} \in \mathcal{S}^{d}:\left\langle Q^{\prime}, Q\right\rangle>0\right.$ for all $\left.Q \in \mathcal{S}_{>0}^{d}\right\}$, meaning that $\mathcal{S}_{>0}^{d}$ is a self-dual cone, we have $F \in \mathcal{S}_{>0}^{d}$.

Because of the preceding proposition, minimizing the linear function $I_{\mathcal{D}}$, hence $\langle F, \cdot\rangle$, over all $\mathrm{PQFs}$ with a fixed determinant $D$ is geometrically equivalent to finding the unique $\mathrm{PQF}$ on the boundary of $\mathbf{D}_{D}$ - the determinant- $D$-surface- that has a supporting hyperplane with normal $F$ (see Proposition 9.7). Thus we derive the following proposition (see Proposition 8.2.2 of [62] for a detailed proof).

Proposition 10.3. A linear function $f(\cdot)=\langle F, \cdot\rangle$ with $F \in \mathcal{S}_{>0}^{d}$ has a unique minimum on the determinant-D-surface. Its value is $d \sqrt[d]{D \operatorname{det} F}$ and the minimum is attained at the $P Q F \sqrt[d]{D \operatorname{det} F} F^{-1}$.

Now we can plug Propositions 10.1 and 10.3 together. This yields a local lower bound for the covering density of a PQF. 
Proposition 10.4. Let $\mathcal{D}$ be a Delone triangulation. Let $Q$ be a $P Q F$ for which $\mathcal{D}$ is a refinement of $\operatorname{Del}(Q)$. Then we have a lower bound for the covering density of $Q$ :

$$
\Theta(Q) \geq \Theta_{*}(\mathcal{D})=\sqrt{\left(\frac{d}{d+1}\right)^{d} \operatorname{det} F} \cdot \kappa_{d},
$$

where $F$ is the positive definite matrix given by the equation $I_{\mathcal{D}}(\cdot)=\langle F, \cdot\rangle$. Here and in what follows we denote the local lower bound for the Delone triangulation $\mathcal{D}$ by $\Theta_{*}(\mathcal{D})$.

Proof. By Proposition $10.3, I_{\mathcal{D}}=\langle F, \cdot\rangle$ has the unique minimum $d \sqrt[d]{D \operatorname{det} F}$ on the determinant- $D$-surface. Using this with $D=\operatorname{det} Q$ and applying Proposition 10.1 we get the lower bound $\Theta_{*}(\mathcal{D})$ for $\Theta(Q)$, because of

$$
\begin{aligned}
\left(\frac{\Theta(Q)}{\kappa_{d}}\right)^{2} & =\frac{\mu(Q)^{d}}{\operatorname{det} Q} \geq \frac{\left(I_{\mathcal{D}}(Q) /(d+1)\right)^{d}}{\operatorname{det} Q} \\
& \geq \frac{d^{d} \operatorname{det} Q \operatorname{det} F}{(d+1)^{d} \operatorname{det} Q}=\left(\frac{d}{d+1}\right)^{d} \operatorname{det} F
\end{aligned}
$$

For a local lower bound on the lattice packing-covering constant we minimize the linear function $I_{\mathcal{D}}(\cdot)=\langle F, \cdot\rangle$ over all $\mathrm{PQFs}$ with a fixed homogeneous minimum. In analogy to the above, we replace the determinant- $D$-surface (the boundary of $\mathbf{D}_{D}$ ) by the homogeneous-minimum- $\lambda$-surface (the boundary of $\mathbf{M}_{\lambda}$ ).

Proposition 10.5. Let $\lambda$ be a positive number. Let $\mathcal{D}$ be a Delone triangulation and let $Q$ be a $P Q F$ for which $\mathcal{D}$ is a refinement of $\operatorname{Del}(Q)$. Further, let the minimum of the linear function $I_{\mathcal{D}}(\cdot)=\langle F, \cdot\rangle$ on the homogeneous-minimum- $\lambda$-surface be attained at a $P Q F Q_{F, \lambda}$. Then we have a lower bound for the packing-covering constant of $Q$ :

$$
\gamma(Q) \geq 2 \sqrt{\frac{\left\langle F, Q_{F, \lambda}\right\rangle}{(d+1) \lambda}} .
$$

Proof. The PQF $(\lambda(Q) / \lambda) Q_{F, \lambda}$ attains the minimum of $\langle F, \cdot\rangle$ among PQFs in $\mathbf{M}_{\lambda(Q)}$. Applying Proposition 10.1 gives the desired lower bound:

$$
\left(\frac{\gamma(Q)}{2}\right)^{2}=\frac{\mu(Q)}{\lambda(Q)} \geq \frac{\left(I_{\mathcal{D}}(Q) /(d+1)\right)}{\lambda(Q)} \geq \frac{\left\langle F,(\lambda(Q) / \lambda) Q_{F, \lambda}\right\rangle}{(d+1) \cdot \lambda(Q)}=\frac{\left\langle F, Q_{F, \lambda}\right\rangle}{(d+1) \lambda} .
$$

It is difficult in practice to obtain $Q_{F, \lambda}$ and therefore the lower bound of the proposition. Instead, if we minimize $I_{\mathcal{D}}$ on an approximation $\mathbf{M}_{\lambda}^{\mathcal{D}}$ of $\mathbf{M}_{\lambda}$, suitable for $\mathcal{D}$, things become easier. We set

$$
\mathbf{M}_{\lambda}^{\mathcal{D}}=\left\{Q \in \mathcal{S}_{>0}^{d}: Q[v] \geq \lambda \text { for all } \boldsymbol{v} \in \mathbb{Z}^{d} \text { with }[\boldsymbol{0}, \boldsymbol{v}] \in \mathcal{D}\right\} .
$$

Now finding the minimum of $I_{\mathcal{D}}$ on $\mathbf{M}_{\lambda}^{\mathcal{D}}$ reduces to a linear program with finitely many constraints. By looking at (6) it is easy to see that this linear program is bounded from below since every summand is at least $\lambda$. Because of $\mathbf{M}_{\lambda} \subseteq \mathbf{M}_{\lambda}^{\mathcal{D}}$ we obtain the following practically useful lower bound for $\gamma$. 
Proposition 10.6. Let $\lambda$ be a positive number. Let $\mathcal{D}$ be a Delone triangulation and let $Q$ be a $P Q F$ for which $\mathcal{D}$ is a refinement of $\operatorname{Del}(Q)$. Further, let the minimum of the linear function $I_{\mathcal{D}}(\cdot)=\langle F, \cdot\rangle$ on $\mathbf{M}_{\lambda}^{\mathcal{D}}$ be attained at a $P Q F Q_{F, \lambda}^{\mathcal{D}}$. Then we have a lower bound for the packing-covering constant of $Q$ :

$$
\gamma(Q) \geq \gamma_{*}(\mathcal{D})=2 \sqrt{\frac{\left\langle F, Q_{F, \lambda}^{\mathcal{D}}\right\rangle}{(d+1) \cdot \lambda} .}
$$

\section{Computational Results}

In Section 8 we developed algorithms for the solution of the lattice covering and the lattice packing-covering problem. Here we want to demonstrate that these algorithms are not purely of theoretical interest. We implemented the algorithms in $\mathrm{C}++$, using the package MAXDET ${ }^{3}$ of $\mathrm{Wu}$, Vandenberghe and Boyd and the package $\operatorname{lrs}^{4}$ of Avis as subroutines. The interested reader can download the implementation from our web page [58].

With the implemented algorithm we are able to determine the solution of the lattice covering problem and the lattice packing-covering problem in dimensions $d=1, \ldots, 5$. This hereby reproduced the known results. For dimension $d=5$ this computation on a $2 \mathrm{GHz}$ Intel Pentium computer took less than 90 minutes. By our computations we get a conjectural list of approximations of all locally optimal solutions. In Section 11.1 we give some details.

More importantly, with the help of a heuristic method, which we describe in Section 11.2, this approach produces interesting new lattices in dimensions $d=6$ which give better coverings and packing-coverings than previously known ones. We analyze them in Section 11.3 and in Section 11.4 where we give rigorous proofs of some facts concerning these lattices which we found experimentally. This analysis turned out to be fruitful: The new six-dimensional lattices were our starting point for finding new best-known covering lattices in dimensions 7 and 8 . We describe the seven-dimensional case in Section 11.5. The eight-dimensional case is explained in [57].

\subsection{Dimensions $1, \ldots, 5$}

We described the solutions to both problems in Sections 3 and 4 for the one-, two-, and three-dimensional cases. Since in these cases there is only one type of Delone triangulation, these results are computationally rather trivial.

The four-dimensional case requires more work because there are three inequivalent Delone triangulations in dimension 4. Baranovskii [7] and independently Dickson [29] found all three inequivalent locally optimal solutions to the lattice covering problem in dimension 4. Earlier Delone and Ryshkov showed that the lattice $A_{4}^{*}$ gives the best

\footnotetext{
${ }^{3}$ http: // www. stanford. edu/ boyd/MAXDET.html.

${ }^{4}$ http://cgm.cs.mcgill.ca/ avis/C/Irs.html.
} 
four-dimensional lattice covering without determining all locally optimal solutions. We describe their approach in Section 11.2.

For the lattice packing-covering problem the four-dimensional case was resolved by Horváth [43]. He shows that there exist, as in the covering case, three isolated, locally optimal solutions - one for each Delone triangulation. It is interesting that Horváth's optimal packing-covering lattice $\mathrm{Ho}_{4}$ does not belong to the family of root lattices and their duals. An associated PQF is

$$
Q_{\mathrm{Ho}_{4}}=\left(\begin{array}{rrrr}
2 & 1 & -1 & -1 \\
1 & 2 & -1 & -1 \\
-1 & -1 & 2 & 0 \\
-1 & -1 & 0 & 2
\end{array}\right)+\frac{1}{3} \sqrt{3}\left(\begin{array}{rrrr}
4 & 1 & -2 & -2 \\
1 & 4 & -2 & -2 \\
-2 & -2 & 4 & 1 \\
-2 & -2 & 1 & 4
\end{array}\right) .
$$

The first summand, associated to the best packing lattice $\mathrm{D}_{4}$, lies on an extreme ray of the secondary cone belonging to $Q_{\mathrm{Ho}_{4}}$.

In a series of papers Ryshkov and Baranovskii solved the five-dimensional lattice covering problem. In [51] Ryshkov introduced the concept of C-types. Two Delone triangulations are of the same $C$-type if their 1-skeletons (the graph consisting of vertices and edges of the triangulation) coincide. He gave an algorithm to find all inequivalent $\mathrm{C}$-types in any given dimension. He computed that there are three inequivalent C-types in dimension 4 and that there are 76 inequivalent $\mathrm{C}$-types in dimension 5. Using this list Baranovskii and Ryshkov enumerated 221 (of 222) inequivalent five-dimensional Delone triangulations in [11]. They described the triangulations in more detail in [12]. In the last paper of the series [53] they showed that the lattice $A_{5}^{*}$ provides the least dense five-dimensional lattice covering. In their proof they do not find all locally optimal lattice coverings. By using estimations (the method explained in Section 11.2 is one of their main tools) they merely show that all local minima exceed the covering density of $\mathrm{A}_{5}^{*}$. As mentioned in Section 5.3, Ryshkov and Baranovskii missed one Delone triangulation. Fortunately, this does not give a thinner lattice covering than $A_{5}^{*}$.

Using our algorithm, and the techniques described in Section 9, to obtain certificates we produced a list of inequivalent locally optimal lattice coverings in dimension 5 . This list is conjecturally complete and gives approximations of the local optima. The computation, including all certificates, takes about 90 minutes on a $2 \mathrm{GHz}$ Intel Pentium computer. We can prove the following theorem rigorously.

Theorem 11.1. In dimension 5 there exist at least 216 and at most 218 inequivalent, local minima of the lattice covering density function $\Theta$, ranging from approximately 2.124286 to approximately 2.757993. All of them are attained in the interior of their secondary cones.

Proof. We take a complete list of 222 inequivalent secondary cones, generated by SCC (see Section 5 and our web page [58]). For each cone, we applied the program coop (covering optimizer), which is based on rma (rigorous MAXDET) and available on our web page [58]. It computes certified bounds for $\Theta$ within the closure of a given secondary cone by solving the determinant maximization problem described in Section 8.1. The bounds for the duality gap described in Section 6 are mathematically rigorous, since we use rational arithmetic only. Solving additional determinant maximization problems as 
described in Section 9.2, the program then tries to obtain certificates for the approximated local optimum to be attained in the interior or on the boundary of the secondary cone. As a result, there are 216 certified local optima attained in the interior of secondary cones and four certified non-optima attained on the boundary. This leaves two cases which might be local optima, attained in the interior of their secondary cones. However, if they are attained on the boundary (and the numerical evidence strongly supports this) then they are not local optima, since the corresponding secondary cones adjacent to the facets in question have a smaller local minimum.

Note that our computations prove rigorously that the PQF of Barnes and Trenerry [16] yields the second best locally optimal lattice covering with a density of approximately 2.230117 .

In 1986 Horváth [44] solved the lattice packing-covering in dimension 5. The proof is about 70 pages long (private communication). As in dimension 4, Horváth's lattice $\mathrm{Ho}_{5}$ is not among previously known ones. An associated PQF is

$$
Q_{\mathrm{Ho}_{5}}=\left(\begin{array}{rrrrr}
2 & 1 & 0 & -1 & -1 \\
1 & 2 & -1 & -1 & 0 \\
0 & -1 & 2 & 0 & 0 \\
-1 & -1 & 0 & 2 & -1 \\
-1 & 0 & 0 & -1 & 2
\end{array}\right)+\frac{1+\sqrt{13}}{6}\left(\begin{array}{rrrrr}
6 & 3 & -2 & -2 & -2 \\
3 & 6 & -2 & -3 & -2 \\
-2 & -2 & 6 & -1 & -1 \\
-2 & -3 & -1 & 6 & 0 \\
-2 & -2 & -1 & 0 & 6
\end{array}\right)
$$

Again, the first summand is associated to the best packing lattice $D_{4}$. It has rank 4 and lies on an extreme ray of the secondary cone belonging to $Q_{\mathrm{Ho}_{5}}$.

By applying the propositions of Section 9 we were able to reproduce Horváth's result and moreover to attain a list of locally optimal solutions. Again this list is conjecturally complete and gives approximations of the local optima. Compared with the covering problem, there are many more secondary cones that contain no locally optimal solution. The computation, including all certificates, also takes about 90 minutes on a $2 \mathrm{GHz}$ Intel Pentium computer. We can prove the following theorem rigorously.

Theorem 11.2. In dimension 5 there exist at least 47 and at most 75 local minima of the lattice packing-covering constant $\gamma$, ranging from approximately 1.449456 to approximately 1.557564. At least 45 of them are attained in the interior of their secondary cones.

Proof. As for the proof of Theorem 11.1, we used the complete list of 222 inequivalent secondary cones, generated by scc. For each cone, we applied the program pacoop (packing-covering optimizer), which is also based on rmd and available on our web page [58]. It computes certified bounds for $\gamma$ within the closure of a given secondary cone by rigorously solving the semidefinite program described in Section 8.2. Again the program tries to obtain certificates for the approximated local optimum to be attained in the interior or on the boundary of the secondary cone. As a result, there are 45 certified local optima attained in the interior of secondary cones and 147 certified non-optima attained on the boundary. There are 30 of 222 remaining cases in which the program pacoop did not give a certificate for the optimum to be attained in the interior or the 
boundary of the secondary cone. However, if all of them are attained on the boundary (and the numerical evidence strongly supports this), then there are two local optima on the common boundary of three inequivalent secondary cones each. Thus there exist at least 47 local optima as claimed.

\subsection{Heuristic Methods}

Before going to dimension 6, we explain one important heuristic method, which is essential for finding the new lattices. One of the biggest problems in finding good lattice coverings or packing-coverings in dimension 6 and higher is that it is not a priori clear which Delone triangulations admit good ones. Solving a determinant maximization problem is a rather time-consuming task and there are many inequivalent Delone triangulations to consider. So a desirable tool is a fast computable lower bound, as described in Section 10.

We view the set of Delone triangulations as an undirected labeled graph. A node represents a Delone triangulation and two nodes are adjacent if their Delone triangulations are bistellar neighbors. Let $\mathcal{D}$ be a Delone triangulation. We label its node by the local lower bound $\Theta_{*}(\mathcal{D})$ or $\gamma_{*}(\mathcal{D})$. We can use the labeling in two different ways.

On the one hand it is clear that if the labeling of a node is large, then the considered Delone triangulation does not admit a good lattice covering and lattice packing-covering respectively. Delone and Ryshkov [28] solved the lattice covering problem in dimension 4 by this method. However, the five-dimensional lattice covering problem cannot be solved in this way. From the 222 inequivalent Delone triangulations there are 20 whose local lower bound is smaller than $\Theta\left(A_{5}^{*}\right)$.

On the other hand we can hope that $\mathcal{D}$ admits a good lattice covering or packingcovering if the local lower bound is small. In dimension 6 the hope that good local lower bounds yield good lattice coverings is partially fulfilled. We report on a typical example: We started from the Delone triangulation of Voronoi's principal form of the first type and took a random walk of length 50 . Then we found a node labeled by $\Theta_{*}(\mathcal{D}) \approx 2.585149$. From this we proceeded by taking a neighboring node having the smallest local lower bound. Repeating this greedy strategy resulted in a node labeled by $\Theta_{*}(\mathcal{D}) \approx 2.318034$. This node is interesting for several conjectural "extremeness" properties. It yields the smallest known local lower bound and it has the largest known number of neighbors, namely 130. As we will see in the next section there exists a locally optimal lattice covering which belongs to this node with covering density of approximately 2.466125 . At present this is the second best known six-dimensional lattice covering which is locally optimal. Furthermore we will see that there exists a locally optimal lattice packingcovering belonging to this node which currently defines the best known six-dimensional lattice packing-covering.

\subsection{New Six-Dimensional Lattices}

We have not been able to solve the lattice covering problem in this dimension. However, we found some new interesting covering lattices. As we reported in Section 3, Ryshkov 
[50] asked what is the first dimension $d$ where $\mathrm{A}_{d}^{*}$ does not give the least dense lattice covering?

A classification of all inequivalent Delone triangulations in dimension 6 is not within reach. So we cannot use our algorithm to find the best lattice covering. Nevertheless we ran it partially to find good lattice coverings. Since there are no other good lattice coverings in the neighborhood of Voronoi's principal form of the first type, we used the heuristic method described in the previous section. In this manner we found about 100 inequivalent secondary cones containing lattice coverings better than $A_{6}^{*}$. Thus, we can give an answer to Ryshkov's question:

Theorem 11.3. Dimension $d=6$ is the smallest dimension where the lattice $\mathrm{A}_{d}^{*}$ does not give the least dense lattice covering.

Proof. This theorem follows from Theorem 11.7 or 11.8 .

Two of the new lattice coverings were strikingly good. Of course, by computational optimization we only got numerical estimates of these coverings.

The now second best-known lattice covering which is locally optimal was quite easy to find: running our heuristic method described above finds this lattice covering in most of the trials. An approximation is given by the PQF

$$
Q_{6}^{c 2} \approx\left(\begin{array}{rrrrrr}
1.9982 & 0.5270 & -0.4170 & -0.5270 & 0.5270 & -1.0541 \\
0.5270 & 1.9982 & -0.4170 & -0.5270 & 0.5270 & -1.0541 \\
-0.4170 & -0.4170 & 2.1082 & -1.0541 & -0.4170 & 0.8341 \\
-0.5270 & -0.5270 & -1.0541 & 1.9982 & -0.5270 & -0.4170 \\
0.5270 & 0.5270 & -0.4170 & -0.5270 & 1.9982 & -1.0541 \\
-1.0541 & -1.0541 & 0.8341 & -0.4170 & -1.0541 & 2.1082
\end{array}\right)
$$

and its covering density is $\Theta\left(Q_{6}^{c 2}\right) \approx 2.466125$. The Delone subdivision is a triangulation and its secondary cone has 130 facets. The local lower bound is approximately 2.318034 .

For a while we thought that this might by the best lattice covering in dimension 6 , but then

$$
Q_{6}^{c 1} \approx\left(\begin{array}{rrrrrr}
2.0550 & -0.9424 & 1.1126 & 0.2747 & -0.9424 & -0.6153 \\
-0.9424 & 1.9227 & -0.5773 & -0.7681 & 0.3651 & -0.3651 \\
1.1126 & -0.5773 & 2.0930 & -0.4934 & -0.5773 & -0.9804 \\
0.2747 & -0.7681 & -0.4934 & 1.7550 & -0.7681 & 0.7681 \\
-0.9424 & 0.3651 & -0.5773 & -0.7681 & 1.9227 & -0.3651 \\
-0.6153 & -0.3651 & -0.9804 & 0.7681 & -0.3651 & 1.9227
\end{array}\right)
$$

with covering density $\Theta\left(Q_{6}^{c 1}\right) \approx 2.464802$ came up. The Delone subdivision is a triangulation and its secondary cone has 100 facets. The local lower bound is approximately 2.322204. After this, we did not find any further lattice covering records in dimension 6 . Furthermore, we did not find a six-dimensional Delone triangulation whose secondary cone has more than 130 facets or whose local lower bound is less than 2.318034 . 
Nevertheless, in the secondary cone of $Q_{6}^{c 2}$ we found the PQF

$$
Q_{6}^{p c} \approx\left(\begin{array}{rrrrrr}
2.0088 & 0.5154 & 0.5154 & -0.5154 & 0.9778 & 0.5154 \\
0.5154 & 2.0088 & 0.5154 & -0.5154 & -0.5154 & -0.9778 \\
0.5154 & 0.5154 & 2.0088 & -0.5154 & -0.5154 & 0.5154 \\
-0.5154 & -0.5154 & -0.5154 & 2.0088 & -0.9778 & -0.5154 \\
0.9778 & -0.5154 & -0.5154 & -0.9778 & 2.0088 & 0.9778 \\
0.5154 & -0.9778 & 0.5154 & -0.5154 & 0.9778 & 2.0088
\end{array}\right),
$$

which gives currently the best known lattice packing-covering in dimension 6 with packing-covering constant $\gamma\left(Q_{6}^{p c}\right) \approx 1.411081$. In the next section we examine these new PQFs in greater detail.

\subsection{Beautification and a Unified View}

Although we found an answer to Ryshkov's question, these results are not fully satisfying. We want to know the exact lattices and prove rigorously that they have a good covering density and that they are locally optimal. Even more important, we want to know an interpretation of why these lattice coverings are good. To accomplish this we collect some more data.

The automorphism group of $\operatorname{Del}\left(Q_{6}^{c 2}\right)$ has order 3840 and the one of $\operatorname{Del}\left(Q_{6}^{c 1}\right)$ has order 240. With the knowledge of the groups we were able to compute the extreme rays of both secondary cones [31]. The secondary cone $\mathbf{C}_{1}=\overline{\boldsymbol{\Delta}\left(\operatorname{Del}\left(Q_{6}^{c 2}\right)\right)}$ has 7, 145, 429 and the cone $\mathbf{C}_{2}=\overline{\Delta\left(\operatorname{Del}\left(Q_{6}^{c 1}\right)\right)}$ has 2, 257, 616 extreme rays. Both contain an extreme ray associated to the lattice $\mathrm{E}_{6}^{*}$ given for example by the $\mathrm{PQF}$

$$
Q_{\mathrm{E}_{6}^{*}}=\left(\begin{array}{rrrrrr}
4 & 1 & 2 & 2 & -1 & 1 \\
1 & 4 & 2 & 2 & 2 & 1 \\
2 & 2 & 4 & 1 & 1 & 2 \\
2 & 2 & 1 & 4 & 1 & 2 \\
-1 & 2 & 1 & 1 & 4 & 2 \\
1 & 1 & 2 & 2 & 2 & 4
\end{array}\right)
$$

After transforming $Q_{6}^{c 1}$ and $Q_{6}^{c 2}$ by integral unimodular transformations we can assume that the Delone triangulations of the two PQFs are refinements of the Delone subdivision $\operatorname{Del}\left(Q_{\mathrm{E}_{6}^{*}}\right)$. This Delone subdivision was investigated in different contexts (see [65], [23], [47] and [9]). We briefly review the main results:

Proposition 11.4. In the star of the origin are 720 full-dimensional six-dimensional Delone polytopes, and the automorphism group of $Q_{\mathrm{E}_{6}^{*}}$ acts transitively on these fulldimensional polytopes. Each polytope is the convex hull of three regular triangles lying in three pairwise orthogonal affine planes. Each polytope has nine vertices, twenty-seven facets, and three different triangulations, where each triangulation consists of nine sixdimensional simplices. The covering density is $\Theta\left(Q_{\mathrm{E}_{6}^{*}}\right)=8 / 9 \sqrt{3} \cdot \kappa_{6} \approx 2.652071$. 
Proof. Except for the possible refining triangulations, all this data is well known, see for example [23, Summary for $\mathrm{E}_{6}^{*}$ ].

To describe the triangulations we introduce coordinates. A Delone polytope of $Q_{\mathrm{E}_{6}^{*}}$ is similar to the polytope $P=\operatorname{conv}\left\{\boldsymbol{u}_{1}, \boldsymbol{u}_{\omega}, \boldsymbol{u}_{\bar{\omega}}, \boldsymbol{v}_{1}, \boldsymbol{v}_{\omega}, \boldsymbol{v}_{\bar{\omega}}, \boldsymbol{w}_{1}, \boldsymbol{w}_{\omega}, \boldsymbol{w}_{\bar{\omega}},\right\}$ where $\boldsymbol{u}_{1}=\boldsymbol{e}_{1}$, $\boldsymbol{u}_{\omega}=-\frac{1}{2} \boldsymbol{e}_{1}+(\sqrt{3} / 2) \boldsymbol{e}_{2}, \boldsymbol{u}_{\bar{\omega}}=-\frac{1}{2} \boldsymbol{e}_{1}-(\sqrt{3} / 2) \boldsymbol{e}_{2}, \boldsymbol{v}_{1}=\boldsymbol{e}_{3}, \boldsymbol{v}_{\omega}=-\frac{1}{2} \boldsymbol{e}_{3}+(\sqrt{3} / 2) \boldsymbol{e}_{4}$, $\boldsymbol{v}_{\bar{\omega}}=-\frac{1}{2} \boldsymbol{e}_{3}-(\sqrt{3} / 2) \boldsymbol{e}_{4}, \boldsymbol{w}_{1}=\boldsymbol{e}_{5}, \boldsymbol{w}_{\omega}=-\frac{1}{2} \boldsymbol{e}_{5}+(\sqrt{3} / 2) \boldsymbol{e}_{6}$ and $\boldsymbol{w}_{\bar{\omega}}=-\frac{1}{2} \boldsymbol{e}_{5}-(\sqrt{3} / 2) \boldsymbol{e}_{6}$. The three possible triangulations are given by the set of nine six-dimensional simplices:

$$
\begin{aligned}
& \mathcal{T}_{\boldsymbol{u}}=\left\{\text { conv vert } P \backslash\left\{\boldsymbol{v}_{z}, \boldsymbol{w}_{z^{\prime}}\right\}: z, z^{\prime} \in\{1, \omega, \bar{\omega}\},\right. \\
& \mathcal{T}_{\boldsymbol{v}}=\left\{\text { conv vert } P \backslash\left\{\boldsymbol{u}_{z}, \boldsymbol{w}_{z^{\prime}}\right\}: z, z^{\prime} \in\{1, \omega, \bar{\omega}\},\right. \\
& \mathcal{T}_{\boldsymbol{w}}=\left\{\text { conv vert } P \backslash\left\{\boldsymbol{u}_{z}, \boldsymbol{v}_{z^{\prime}}\right\}: z, z^{\prime} \in\{1, \omega, \bar{\omega}\}\right\} .
\end{aligned}
$$

To finish the proof one has to show that these sets indeed define triangulations and that they are the only possible triangulations. This can be done by straightforward computation using the facts that the minimal affine dependent subsets of vert $P$ are

$$
\left\{\boldsymbol{v}_{1}, \boldsymbol{v}_{\omega}, \boldsymbol{v}_{\bar{\omega}}, \boldsymbol{w}_{1}, \boldsymbol{w}_{\omega}, \boldsymbol{w}_{\bar{\omega}}\right\},\left\{\boldsymbol{u}_{1}, \boldsymbol{u}_{\omega}, \boldsymbol{u}_{\bar{\omega}}, \boldsymbol{w}_{1}, \boldsymbol{w}_{\omega}, \boldsymbol{w}_{\bar{\omega}}\right\},\left\{\boldsymbol{u}_{1}, \boldsymbol{u}_{\omega}, \boldsymbol{u}_{\bar{\omega}}, \boldsymbol{v}_{1}, \boldsymbol{v}_{\omega}, \boldsymbol{v}_{\bar{\omega}}\right\}
$$

that the facets of $P$ are conv vert $P \backslash\left\{\boldsymbol{u}_{z}, \boldsymbol{v}_{z^{\prime}}, \boldsymbol{w}_{z^{\prime \prime}}\right\}$ where $z, z^{\prime}, z^{\prime \prime} \in\{1, \omega, \bar{\omega}\}$, and by applying the following proposition.

Proposition 11.5 (Proposition 2.2 of [49]). Let $\mathcal{A} \subseteq \mathbb{R}^{d}$ be a finite point set, and let $\mathcal{T}$ be a set of $d$-dimensional simplices with vertices in $\mathcal{A}$. The set $\mathcal{T}$ defines a triangulation of the polytope conv $\mathcal{A}$ if and only if the following two conditions hold:

1. For all $S, S^{\prime} \in \mathcal{T}$ there exists a minimal affine dependency $\sum_{\boldsymbol{a} \in \mathcal{A}} \lambda_{a} \boldsymbol{a}=\mathbf{0}$ with $\sum_{\boldsymbol{a} \in \mathcal{A}} \lambda_{\boldsymbol{a}}=0$ so that $\left\{\boldsymbol{a} \in \mathcal{A}: \lambda_{\boldsymbol{a}}>0\right\} \subseteq S$ and $\left\{\boldsymbol{a} \in \mathcal{A}: \lambda_{\boldsymbol{a}}<0\right\} \subseteq S^{\prime}$.

2. For all $S \in \mathcal{T}$ and for every $(d-1)$-dimensional facet $F$ of $S$ there exists either $a(d-1)$-dimensional facet $F^{\prime}$ of $\operatorname{conv} \mathcal{A}$ with $F \subseteq F^{\prime}$ or there exists another simplex $S^{\prime} \in \mathcal{T} \backslash\{S\}$ also having $F$ as a facet.

Using this information we are able to prove that the PQF $Q_{6}^{c 1}$ is closely related to $Q_{\mathrm{E}_{6}^{*}}$.

Theorem 11.6. The $P Q F Q_{6}^{c 1}$ gives the least dense lattice covering among all $P Q F s$ whose Delone subdivision is a refinement of the Delone subdivision $\operatorname{Del}\left(Q_{\mathrm{E}_{6}^{*}}\right)$.

Proof. Our proof is computational and uses a branch and cut method. We have to show that all secondary cones of a Delone triangulation refining $\operatorname{Del}\left(Q_{\mathrm{E}_{6}^{*}}\right)$ do not contain a PQF with covering density less than $\Theta\left(Q_{6}^{c 1}\right)$. There are 40 full-dimensional Delone polytopes of $Q_{\mathrm{E}_{6}^{*}}$ which cannot be transformed into each other by translations or by the map $\boldsymbol{x} \mapsto-\boldsymbol{x}$. Since each of these Delone polytopes has three possible triangulations, the number of all periodic triangulations refining $\operatorname{Del}\left(Q_{\mathrm{E}_{6}^{*}}\right)$ is $3^{40}$. It is not a priori clear how to distinguish between Delone and non-Delone triangulations, and it is not possible to generate all $3^{40}$ triangulations. We choose a backtracking approach instead.

We arrange partial triangulations in a tree. On every level one of the 40 Delone polytopes is triangulated so that we have $3^{n}$ nodes on the $n$th level. For every node $N$ 
we define the value

$$
\begin{gathered}
\Theta_{N}=\max \left\{\operatorname{det}(Q): Q \in \mathcal{S}_{>0}^{6}, \mathrm{BR}_{L}(Q) \succeq 0 \text { for all simplices } L\right. \\
\text { of partial triangulation } N\} .
\end{gathered}
$$

Obviously, $\Theta_{N}$ is a lower bound for the covering density of any PQF whose Delone subdivision refines the partial triangulation $N$. We can compute a lower bound of this value by solving a determinant maximization problem similar to the one in Section 8.1. Note that this can be done rigorously using rational arithmetic only if we proceed as described in Section 6. If the lower bound is larger than 2.464802, we can cut the tree at this node, since the covering density of $Q_{6}^{c 1}$ is less than 2.464801 (see Theorem 11.8).

This algorithm visits exactly 432 nodes of depth 40 . One of these triangulations equals $\operatorname{Del}\left(Q_{6}^{c 1}\right)$. All the others are equivalent to $\operatorname{Del}\left(Q_{6}^{c 1}\right)$ because the automorphism group of $\operatorname{Del}\left(Q_{6}^{c 1}\right)$, which has order 240, is a subgroup of $\operatorname{Aut}\left(Q_{\mathrm{E}_{6}^{*}}\right)$ and the order of $\operatorname{Aut}\left(Q_{\mathrm{E}_{6}^{*}}\right)$ equals $103,680=432 \cdot 240$.

This computational proof takes about 2 weeks on a $2 \mathrm{GHz}$ Intel Pentium computer. The source code e6d.cc is available from our web page [58] as part of the package rma.

Knowledge of the automorphism groups of $\operatorname{Del}\left(Q_{6}^{c 1}\right)$ and $\operatorname{Del}\left(Q_{6}^{c 2}\right)$ also enables us to give a unified view on both lattices. We have

$$
\operatorname{Aut}\left(\operatorname{Del}\left(Q_{6}^{c 1}\right)\right) \subseteq \operatorname{Aut}\left(\operatorname{Del}\left(Q_{6}^{c 2}\right)\right) \subseteq \operatorname{Aut}\left(Q_{\mathrm{E}_{6}^{*}}\right) .
$$

The automorphism group $\operatorname{Aut}\left(\operatorname{Del}\left(Q_{6}^{c 2}\right)\right)$ turns out to be the subgroup of $\operatorname{Aut}\left(Q_{\mathrm{E}_{6}^{*}}\right)$ stabilizing the minimal vectors $\pm \boldsymbol{e}_{1}$, and $\operatorname{Aut}\left(\operatorname{Del}\left(Q_{6}^{c 1}\right)\right)$ is the intersection of the two subgroups of $\operatorname{Aut}\left(Q_{\mathrm{E}_{6}^{*}}\right)$ stabilizing the minimal vectors $\pm \boldsymbol{e}_{1}$ and $\pm \boldsymbol{e}_{2}$ respectively.

The subspace $\mathbf{I}_{1}$ of all quadratic forms invariant under the group $\operatorname{Aut}\left(\operatorname{Del}\left(Q_{6}^{c 2}\right)\right)$ is spanned by the PQFs $Q_{\mathrm{E}_{6}^{*}}$ and $R_{1}$ (see below). At the same time, $\mathbf{I}_{1} \cap \mathbf{C}_{1}$ is a cone with extreme rays $Q_{\mathrm{E}_{6}^{*}}$ and $R_{1}$. By Proposition 9.2, $Q_{6}^{c 2}$ has to lie in cone $\left\{Q_{\mathrm{E}_{6}^{*}}, R_{1}\right\}$. The subspace $\mathbf{I}_{2}$ of all quadratic forms invariant under the group $\operatorname{Aut}\left(\operatorname{Del}\left(Q_{6}^{c 1}\right)\right)$ is fourdimensional. The cone $\mathbf{I}_{2} \cap \mathbf{C}_{2}$ has six extreme rays $Q_{\mathrm{E}_{6}^{*}}, R_{2}, \ldots, R_{6}$, where

$$
\begin{array}{rlrl}
R_{1} & =\left(\begin{array}{rrrrrr}
12 & 3 & 6 & 6 & -3 & 3 \\
3 & 7 & 4 & 4 & 3 & 2 \\
6 & 4 & 8 & 3 & 1 & 4 \\
6 & 4 & 3 & 8 & 1 & 4 \\
-3 & 3 & 1 & 1 & 7 & 3 \\
3 & 2 & 4 & 4 & 3 & 7
\end{array}\right), & R_{2}=\left(\begin{array}{rrrrrr}
0 & 0 & 0 & 0 & 0 & 0 \\
0 & 5 & 2 & 2 & 3 & 1 \\
0 & 2 & 4 & 0 & 2 & 2 \\
0 & 2 & 0 & 4 & 2 & 2 \\
0 & 3 & 2 & 2 & 5 & 3 \\
0 & 1 & 2 & 2 & 3 & 5
\end{array}\right), \\
R_{3}=\left(\begin{array}{rrrrrr}
6 & 4 & 4 & 4 & 0 & 2 \\
4 & 11 & 6 & 6 & 5 & 3 \\
4 & 6 & 8 & 3 & 3 & 4 \\
4 & 6 & 3 & 8 & 3 & 4 \\
0 & 5 & 3 & 3 & 7 & 4 \\
2 & 3 & 4 & 4 & 4 & 7
\end{array}\right), & R_{4}=\left(\begin{array}{rrrrrr}
3 & 2 & 2 & 2 & 0 & 1 \\
2 & 3 & 2 & 2 & 1 & 1 \\
2 & 2 & 4 & 1 & 1 & 2 \\
2 & 2 & 1 & 4 & 1 & 2 \\
0 & 1 & 1 & 1 & 3 & 2 \\
1 & 1 & 2 & 2 & 2 & 4
\end{array}\right),
\end{array}
$$




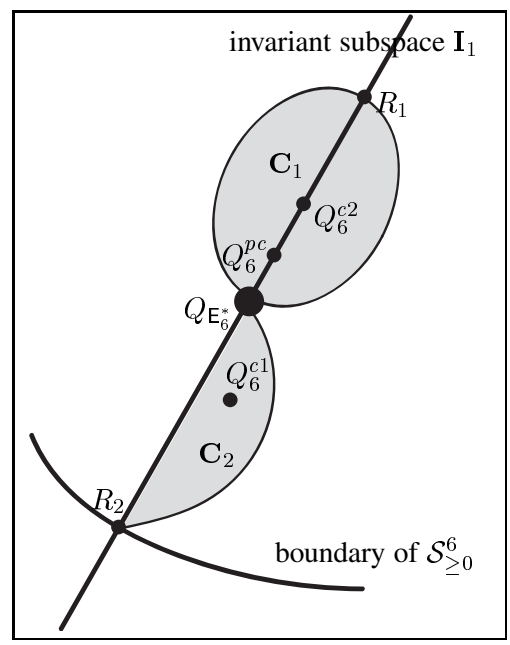

Fig. 5. The graph of two-dimensional Delone triangulations.

$$
R_{5}=\left(\begin{array}{rrrrrr}
7 & 3 & 4 & 4 & -1 & 2 \\
3 & 17 & 8 & 8 & 9 & 4 \\
4 & 8 & 12 & 3 & 5 & 6 \\
4 & 8 & 3 & 12 & 5 & 6 \\
-1 & 9 & 5 & 5 & 13 & 7 \\
2 & 4 & 6 & 6 & 7 & 12
\end{array}\right), \quad R_{6}=\left(\begin{array}{llllll}
9 & 6 & 6 & 6 & 0 & 3 \\
6 & 9 & 6 & 6 & 3 & 3 \\
6 & 6 & 8 & 4 & 2 & 4 \\
6 & 6 & 4 & 8 & 2 & 4 \\
0 & 3 & 2 & 2 & 5 & 3 \\
3 & 3 & 4 & 4 & 3 & 6
\end{array}\right)
$$

Note that $R_{2}$ lies in $\mathbf{I}_{1}$. Altogether, this yields the "picture" in dimension 21 given in Fig. 5.

Let us finally try to find the exact coordinates of the PQFs. This is easy for $Q_{6}^{c 2}$. We know that we can scale $Q_{6}^{c 2}$ so that $Q_{6}^{c 2}=Q_{\mathrm{E}_{6}^{*}}+x R_{1}$, for some $x \in \mathbb{R}_{\geq 0}$. Now, the exact finding of $x$ boils down to finding roots of a univariate polynomial: we have to minimize the function $x \mapsto \mu\left(Q_{\mathrm{E}_{6}^{*}}+x R_{1}\right)^{d} / \operatorname{det}\left(Q_{\mathrm{E}_{6}^{*}}+x R_{1}\right)$ where we know, because of the approximate solution, that $\mu\left(Q_{\mathrm{E}_{6}^{*}}+x R_{1}\right)$ is a polynomial for all points in a sufficiently small neighborhood of the exact $x$. This leads to the Ansatz

$$
Q_{6}^{c 2}=Q_{\mathrm{E}_{6}^{*}}+\frac{\sqrt{1057}-1}{88} R_{1}
$$

Now we can use the tools we introduced in Section 9 to prove rigorously that this Ansatz works.

Theorem 11.7. The $P Q F Q_{6}^{c 2}$ gives a locally optimal lattice covering. Its covering density is

$$
\Theta\left(Q_{6}^{c 2}\right)=\frac{\sqrt{1124895337698 \sqrt{1057}-33713139497730}}{3543122} \cdot \kappa_{6} \approx 2.466121650 .
$$


Proof. Our proof is again computational. We provide the MAGMA program check_q6c2.m at the arXiv.org e-print archive. To access it, download the source files for the paper math. MG / 0403272 . Since it uses rational arithmetic only, the proof is rigorous.

We describe the steps. First we compute the Delone subdivision of $Q_{6}^{c 2}$ : We compute the Delone subdivisions of the three PQFs $Q_{1}=\frac{9}{10} Q_{\mathrm{E}_{6}^{*}}+\frac{3}{10} R_{1}, Q_{2}=\frac{11}{10} Q_{\mathrm{E}_{6}^{*}}+\frac{3}{10} R_{1}$ and $Q_{3}=\frac{11}{10} Q_{\mathrm{E}_{6}^{*}}+\frac{5}{10} R_{1}$. Then we check that they coincide and that it turns out to be a triangulation. Then we show that $Q_{6}^{c 2} \in \operatorname{conv}\left\{Q_{1}, Q_{2}, Q_{3}\right\}$ so that $\operatorname{Del}\left(Q_{6}^{c 2}\right)=\operatorname{Del}\left(Q_{1}\right)$. Now we can compute the circumradii of all the Delone simplices by formula (3) in the proof of Proposition 7.1. This gives the value $\Theta\left(Q_{6}^{c 2}\right)$. Finally we prove that $Q_{6}^{c 2}$ gives a locally optimal lattice covering using the criterion of Proposition 9.9. We compute the gradients $g_{L}$ for all simplices $L \in \operatorname{Del}\left(Q_{6}^{c 2}\right)$ achieving maximum circumradius. Summing them up yields a multiple of $-\left(Q_{6}^{c 2}\right)^{-1}$.

This computational proof takes about 1 minute on a $2 \mathrm{GHz}$ Intel Pentium computer.

What is the general pattern behind the beautification process? Let $Q$ be a locally optimal lattice covering with Delone triangulation $\mathcal{D}$. We use the symmetry of $\mathcal{D}$ to find the subspace in which $Q$ lies. This reduces the number of involved variables. The simplices of the Delone triangulation which have circumradius 1 give equality constraints. Then we maximize the determinant of the quadratic forms lying in the subspace subject to the equality constraints. For this optimization problem, which involves only algebraic equations, we can use Gröbner basis techniques.

Unfortunately, we were not able to solve the corresponding algebraic equations for $Q_{6}^{c 1}$ so we have to be satisfied with an approximation.

Theorem 11.8. The covering density of the $P Q F Q_{6}^{c 1}$ is at most 2.464801 .

Proof. The covering density of the PQF $Q_{\mathrm{E}_{6}^{*}}+x R_{2}+y R_{3}+z R_{4}$ with $x=$ $0.15266328480099, y=0.32884740614948$ and $z=0.13827491241153$ is smaller than 2.464801. For a computational proof of this fact we provide the MAGMA program check_q6 $1 . \mathrm{m}$, available from the source files of the paper math.MG/0403272 at the arXiv.org e-print archive. The steps are similar to the first steps of the proof of Theorem 11.7.

On the basis of Theorem 11.6 and our extensive computational experiments we make the following conjecture.

Conjecture 11.9. The $P Q F Q_{6}^{c 1}$ provides the unique least dense lattice covering in dimension 6.

Using a similar and more successful beautification process for $Q_{6}^{p c}$, we make the Ansatz

$$
Q_{6}^{p c}=Q_{\mathrm{E}_{6}^{*}}+\frac{\sqrt{798}-18}{79} R_{1}
$$


Theorem 11.10. $Q_{6}^{p c}$ is a locally optimal solution to the lattice packing-covering problem, lying in the interior of its secondary cone. Its lattice packing covering constant is

$$
\gamma\left(Q_{6}^{p c}\right)=2 \sqrt{2 \sqrt{798}-56} \approx 1.411081242 .
$$

Proof. This is similar to the proof of Theorem 11.7. We provide the MAGMA program check_q6pc.m, available from the source files of the paper math.MG / 0403272 at the arXiv.org e-print archive.

\subsection{Dimension 7}

After analyzing the six-dimensional case, we got a feeling of where we have to search for good seven-dimensional lattice coverings. We took $Q_{\mathrm{E}_{7}^{*}}$ and a lattice vector which is a longest vector of the shortest vectors in the cosets $\mathbb{Z}^{d} / 2 \mathbb{Z}^{d}$. We computed the stabilizer group of this vector and the invariant subspace of this group. By perturbing $Q_{\mathrm{E}_{7}^{*}}$ in this subspace randomly, we found a PQF whose Delone subdivision is a triangulation. We solved the determinant maximization problem of Section 8.1 which belongs to this Delone triangulation and found the PQF

$$
Q_{7}^{c}=\left(\begin{array}{rrrrrrr}
12 & 1 & 1 & 1 & 1 & 1 & 5 \\
1 & 12 & 1 & 1 & 1 & 1 & 5 \\
1 & 1 & 12 & 1 & 1 & 1 & 5 \\
1 & 1 & 1 & 12 & 1 & 1 & 5 \\
1 & 1 & 1 & 1 & 12 & 1 & 5 \\
1 & 1 & 1 & 1 & 1 & 12 & -6 \\
5 & 5 & 5 & 5 & 5 & -6 & 14
\end{array}\right) .
$$

We are quite surprised that this PQF has rational entries.

Theorem 11.11. $\quad Q_{7}^{c}$ is a locally optimal solution to the lattice covering problem, lying in the interior of its secondary cone. Its inhomogeneous minimum is $\mu=\frac{15}{2}$, its determinant is $\operatorname{det} Q_{7}^{c}=2 \cdot 11^{6}$, so that $\Theta\left(Q_{7}^{c}\right) \approx 2.900024$.

Proof. Again, this is similar to the proof of Theorem 11.7. We provide the MAGMA program check_q7 c.m, available from the source files of the paper math.MG / 0403272 at the arXiv. org e-print archive.

\section{Acknowledgments}

We thank Mathieu Dutour for the simplified formulation of the optimization problem in Section 8.2, Jörg Rambau for pointing out the reference to Proposition 11.5, Francisco Santos for helpful discussions and Tyrrell B. McAllister for improving the grammar of our text. In particular we thank one of the anonymous referees for the detailed report with many helpful comments and suggestions on a previous version. 


\section{References}

1. V. Alexeev, Complete moduli in the presence of semiabelian group action, Ann. Math. 155 (2002), 611708.

2. M.M. Anzin, On the density of a lattice covering for $n=11$ and $n=14$, Russian Math. Surveys 57 (2002), 407-409, translation from Uspekhi Mat. Nauk 57, No. 2(344) (2002), 187-188.

3. T.M. Apostol and M.A. Mnatsakanian, Sums of squares of distances in $m$-space, Amer. Math. Monthly 110 (2003), 516-526.

4. R.P. Bambah, Lattice coverings with four-dimensional spheres, Math. Proc. Cambridge Philos. Soc. 50 (1954), 203-208.

5. R.P. Bambah, On lattice coverings by spheres, Proc. Nat. Acad. Sci. India 20 (1954), 25-52.

6. R.P. Bambah and N.J.A. Sloane, On a problem of Ryshkov concerning lattice coverings, Acta Arith. 42(1) (1982), 107-109.

7. E.P. Baranovskii, Local density minima of a lattice covering of a four-dimensional Euclidean space by equal spheres, Soviet Math. Dokl. 6 (1965), 1131-1133, translation from Dokl. Akad. Nauk SSSR 164 (1965), 13-15.

8. E.P. Baranovskii, Local minima of the density of a lattice covering of four-dimensional Euclidean space by equal balls, Siberian Math. J. 7 (1966), 779-798, translation from Sibirsk. Mat. Zh. 7 (1966), 974-1001.

9. E.P. Baranovskii, Partition of Euclidean spaces into $L$-polytopes of certain perfect lattices, Proc. Steklov Inst. Math. 196 (1992), 29-51, translation from Trudy Mat. Inst. Steklov 196 (1991), 27-46.

10. E.P. Baranovskii, The perfect lattices $\Gamma\left(\mathfrak{A}_{n}\right)$, and the covering density of $\Gamma\left(\mathfrak{A}_{9}\right)$, European J. Combin. 15(4) (1994), 317-323.

11. E.P. Baranovskii and S.S. Ryshkov, Primitive five-dimensional parallelohedra, Soviet Math. Dokl. 14 (1973), 1391-1395, translation from Dokl. Akad. Nauk SSSR 212 (1975), 532-535.

12. E.P. Baranovskii and S.S. Ryshkov, The combinatorial-metric structure of $L$-partitions of general fivedimensional lattices, Soviet Math. Dokl. 16 (1975), 47-51, translation from Dokl. Akad. Nauk SSSR 220 (1975), 265-268.

13. E.P. Baranovskii and S.S. Ryshkov, Classical methods in the theory of lattice packings, Russian Math. Surv. 34(4) (1979), 1-68, translation from Uspekhi Mat. Nauk 34, No. 4(208), (1979), 3-63.

14. E.S. Barnes, The covering of space by spheres, Canad. J. Math. 8 (1956), 293-304.

15. E.S. Barnes and T.J. Dickson, Extreme coverings of $n$-space by spheres, J. Austral. Math. Soc. 7 (1967), 115-127, corrigendum, ibid. 8 (1968), 638-640.

16. E.S. Barnes and D.W. Trenerry, A class of extreme lattice-coverings of $n$-space by spheres, J. Austral. Math. Soc. 14 (1972), 247-256.

17. M. Berger, Geometry I, Springer-Verlag, Berlin, 1987.

18. M.N. Bleicher, Lattice coverings of $n$-space by spheres, Canad. J. Math. 14 (1962), 632-650.

19. K. Böröczky, Closest packing and loosest covering of the space with balls, Studia Sci. Math. Hungar. 21 (1986), 79-89.

20. K.Q. Brown, Voronoi diagrams from convex hulls, Inform. Process. Lett. 9 (1979), 223-228.

21. G.J. Butler, Simultaneous packing and covering in Euclidean space, London Math. Soc. 25 (1972), 721 735.

22. J.H. Conway and N.J.A. Sloane, Sphere Packings, Lattices and Groups, Springer-Verlag, New York, 1988.

23. J.H. Conway and N.J.A. Sloane, The cell structures of certain lattices, in Miscellanea Mathematica, Springer-Verlag, Berlin, 1991, pp. 71-107.

24. H.S.M. Coxeter, Extreme forms, Canad. J. Math. 3 (1951), 391-441.

25. B.N. Delone, Sur la partition régulière de l'espace a 4 dimensions, Izv. Akad. Nauk SSSR Otdel. Fiz.-Mat. Nauk (1929), 79-110 and 145-164.

26. B.N. Delone, The geometry of positive quadratic forms (in Russian), Uspekhi Mat. Nauk 4 (1938), 102-164.

27. B.N. Delone, N.P. Dolbilin, S.S. Ryshkov and M.I. Stogrin, A new construction of the theory of lattice coverings of an $n$-dimensional space by congruent balls, Math. USSR-Izv. 4 (1970), 293-302.

28. B.N. Delone and S.S. Ryshkov, Solution of the problem on the least dense lattice covering of a 4dimensional space by equal spheres, Soviet Math. Dokl. 4 (1963), 1333-1334, translation from Dokl. Akad. Nauk SSSR 152 (1963), 523-224.

29. T.J. Dickson, The extreme coverings of 4-space by spheres, J. Austral. Math. Soc. 7 (1967), 490-496.

30. M.M. Deza and M. Laurent, Geometry of Cuts and Metrics, Springer-Verlag, Berlin, 1997. 
31. M. Dutour and F. Vallentin, Some six-dimensional rigid forms, to appear in Proceedings of Voronoi Conference on Analytic Number Theory and Spatial Tessellations, 8 pages, math. MG / 0401191.

32. H. Edelsbrunner and R. Seidel, Voronoi diagrams and arrangements, Discrete Comput. Geom. 1 (1986), $25-44$.

33. P. Engel, New investigations of parallelohedra in $\mathbb{R}^{d}$, in Voronoi's Impact on Modern Science, Institute of Mathematics of the National Academy of Sciences of Ukraine, Kiev, translation from Proc. Math Ukraine "Voronoi's Impact on Modern Science" 21 (1998), 22-60.

34. P. Engel, The contraction types of parallelohedra in $\mathbb{E}^{5}$, Acta Cryst. Sect. A 56 (2000), 491-496.

35. P. Engel and V.P. Grishukhin, There are exactly $222 L$-types of primitive five-dimensional lattices, European J. Combin. 23 (2002), 275-279.

36. L. Fejes Tóth, Close packing and loose covering with balls, Publ. Math. Debrecen 23 (1976), 324-326.

37. L. Few, Covering space by spheres, Mathematika 3 (1956), 136-139.

38. A.F. Gameckii, On the theory of covering Euclidean $n$-space by equal spheres, Soviet Math.Dokl.3(1962), 1410-1414, translation from Dokl. Akad. Nauk SSSR 146 (1962), 991-994.

39. A.F. Gameckii, The optimality of Voronoi's lattice of first type among lattices of first type of arbitrary dimension, Soviet Math. Dokl. 4 (1963), 1014-1016, translation from Dokl. Akad. Nauk SSSR 151 (1963), 482-484.

40. I.M. Gel'fand, M.M. Kapranov and A.V. Zelevinsky, Discriminants, Resultants, and Multidimensional Determinants, Birkhäuser, Boston, MA, 1994.

41. P.M. Gruber and C.G. Lekkerkerker, Geometry of Numbers, North-Holland, Amsterdam, 1987.

42. P.M. Gruber and S.S. Ryshkov, Facet-to-facet implies face-to-face, European J. Combin. 10(1) (1989), $83-84$.

43. J. Horváth, On close lattice packing of unit spheres in the space $E^{n}$, Proc. Steklov Inst. Math. 152 (1982), 237-254, translation from Trudy Mat. Inst. Steklova 152 (1980), 216-231.

44. J. Horváth, Several problems of $n$-dimensional discrete geometry, Ph.D. thesis, Steklov Institute of Mathematics, 1986.

45. R. Kershner, The number of circles covering a set, Amer. J. Math. 61 (1939), 665-671.

46. J.C. Lagarias and P.A.B. Pleasants, Local complexity of Delone sets and crystallinity, Canad. Math. Bull. 45 (2002), 634-652.

47. R.V. Moody and J. Patera, Voronoi domains and dual cells in the generalized kaleidoscope with applications to root and weight lattices, Canad. J. Math. 47(3) (1995), 573-605.

48. Y. Nesterov and A. Nemirovskii, Interior-Point Polynomial Algorithms in Convex Programming, vol. 13, Society for Industrial and Applied Mathematics (SIAM), Philadelphia, PA, 1994.

49. J. Rambau, Triangulations of cyclic polytopes and higher Bruhat orders, Mathematika 44 (1997), 162-194.

50. S.S. Ryshkov, Effectuation of a method of Davenport in the theory of coverings, Soviet Math. Dokl. 8 (1967), 865-867, translation from Dokl. Akad. Nauk SSSR 175 (1967), 303-305.

51. S.S. Ryshkov, $C$-types of $n$-dimensional parallelohedra, Soviet Math. Dokl. 14 (1973), 1314-1318, translation from Dokl. Akad. Nauk SSSR 212 (1973), 46-49.

52. S.S. Ryshkov, Density of an $(r, R)$-system, Math. Notes 16 (1974), 855-858, translation from Mat.Zametki 1 (1974), 447-454.

53. S.S. Ryshkov and E.P. Baranovskii, Solution of the problem of the least dense lattice covering of fivedimensional space by equal spheres, Soviet Math. Dokl. 16 (1975), 586-590, translation from Dokl. Akad. Nauk SSSR 222 (1975), 39-42.

54. S.S. Ryshkov and E.P. Baranovskii, $C$-types of $n$-dimensional lattices and 5-dimensional primitive parallelohedra (with application to the theory of coverings), Proc. Steklov Inst. Math. (4) (1978), 1-140, translation of Trudy Mat. Inst. Steklov 137 (1976), 1-131.

55. W. Scharlau and H. Opolka, From Fermat to Minkowski, Springer-Verlag, New York, 1985.

56. R. Schneider, Convex Bodies: The Brunn-Minkowski Theory, Cambridge University Press, Cambridge, 1993.

57. A. Schürmann and F. Vallentin, Local covering optimality of lattices: Leech lattice versus root lattice $E_{8}$, Internat. Math. Res. Notices, 2005 (32) (2005), 1937-1955.

58. A. Schürmann and F. Vallentin, Geometry of lattices and algorithms, 2005, http://www.math.uni-magdeburg.de/lattice_geometry/.

59. W.D. Smith, Studies in computational geometry motivated by mesh generation, Ph.D. thesis, Department of Applied Mathematics, Princeton University, 1988. 
60. M.I. Stogrin, Regular Dirichlet-Voronoi partitions for the second triclinic group, Proc. Steklov Inst. Math. 123 (1975), 1-116, translation from Trudy Mat. Inst. Steklov 123 (1973), 1-128.

61. K.C. Toh, Primal-dual path-following algorithms for determinant maximization problems with linear matrix inequalities, Comput. Optim. Appl. 14(3) (1999), 309-330.

62. F. Vallentin, Sphere coverings, lattices, and tilings (in low dimensions), Ph.D. thesis, Center for Mathematical Sciences, Munich University of Technology, 2003, http://tumb1.biblio. tu-muenchen. de/publ/diss/ma/2003/vallentin.html.

63. L. Vandenberghe, S. Boyd and S.P. Wu, Determinant maximization with linear matrix inequality constraints, SIAM J. Matrix Anal. Appl. 19(2) (1998), 499-533.

64. G.F. Voronoi, Nouvelles applications des paramètres continus à la théorie des formes quadratiques. Deuxiéme Mémoire. recherches sur les parallélloedres primitifs., J. Reine Angew. Math. 134 (1908), 198-287, and 136 (1909), 67-181.

65. R.T. Worley, The Voronoi region of $E_{6}^{*}$, J. Austral. Math. Soc. Ser. A 43(2) (1987), 268-278.

66. C. Zong, From deep holes to free planes, Bull. Amer. Math. Soc. 39 (2002), 533-555.

Received March 29, 2004, and in revised form June 22, 2005. Online publication October 12, 2005. 